\title{
INDEX THEORY AND NON-COMMUTATIVE GEOMETRY I. HIGHER FAMILIES INDEX THEORY
}

\author{
MOULAY-TAHAR BENAMEUR AND JAMES L. HEITSCH
}

\begin{abstract}
We prove an index theorem for foliated manifolds. We do so by constructing a push forward map in cohomology for a K-oriented map from an arbitrary manifold to the space of leaves of an oriented foliation, and by constructing a Chern-Connes character from the K-theory of the compactly supported smooth functions on the holonomy groupoid of the foliation to the Haefliger cohomology of the foliation. Combining these with the Connes-Skandalis topological index map and the classical Chern character gives a commutative diagram from which the index theorem follows immediately.
\end{abstract}

\section{Contents}

1. Introduction

2. Notation and Review

3. Traces

4. The algebraic Chern character

5. Push forward maps

6. The index theorem

\section{INTRODUCTION}

This is the first in a series of papers on the different approaches to the higher index and Lefschetz theorems for foliations. In this paper, we give an alternative proof of the cohomological index theorem for families of elliptic operators defined along the leaves of a foliation of a compact manifold obtained in [HL99]. We do so by constructing a push forward map in cohomology for a K-oriented map from an arbitrary manifold to the space of leaves of the foliation, and by constructing a Chern-Connes character from the K-theory of the compactly supported smooth functions on the holonomy groupoid of the foliation to the Haefliger cohomology of the foliation. Combining these with the Connes-Skandalis topological index map and the classical Chern character gives a commutative diagram completely analogous to the commutative diagram of classical families index theory. As in the classical case, this immediately gives the cohomological index theorem obtained in [HL99].

In [CS84] and [Con81], Connes and Skandalis proved a families index theorem for foliations. Their theorem and its proof are algebraic in nature, showing that two push forward maps in K-theory are the same. Our constructions are more geometric and we get computable invariants in Haefliger cohomology. In addition, these invariants are more easily related to the geometry and topology of the foliation. The algebraic approach to invariants for non-commutative spaces is in some sense more fully developed than the geometric. It is our contention that the further development of the geometric approach will lend deep insight into these invariants. We expect that the application of the results here will give a much fuller understanding of the relationship of the index theory of leafwise operators with the geometry and topology of the foliation. Such insight is also likely to have applications to the Novikov conjecture, one of the central problems in topology. 
In [A75], Atiyah identified the index of an elliptic differential operator $D$ on a compact manifold with the trace in an associated von Neumann algebra of a primary $K$-theory invariant of the operator. This latter fundamental invariant lives in the $K$-theory of the $C^{*}$-algebra associated with the fundamental group of $M$ and is a generalized index of the lifted operator $\widetilde{D}$ of $D$ to the universal cover $\widetilde{M}$. The invariance of $\widetilde{D}$ under the deck transformations insures that this deeper index class is well defined even though $\widetilde{M}$ is non-compact in general. This construction led to many deep results in the topological and harmonic analysis of discrete groups, see for instance [BC84, BCH90, CM91, HK99, K88]. Atiyah's covering index theorem was generalized by Connes and Moscovici to higher traces in [CM91], giving a cyclic cohomology proof of the Novikov conjecture for hyperbolic groups.

In [Con79, Con81], Connes extended this construction to leafwise elliptic operators on compact foliated manifolds which are endowed with a holonomy invariant measure. To do so he replaced the lifting and deck transformations by a lifting to the holonomy covers of the leaves invariant under the natural action of the holonomy groupoid. Moreover, he defined an analytic index map from the K-theory of the tangent bundle to the foliation to the K-theory of the $\mathrm{C}^{*}$ algebra of the foliation, which plays the role of the K-theory of the space of leaves. In [CS84], Connes and Skandalis defined a push forward map in K-theory for any K-oriented map from a manifold to the space of leaves of a foliation of a compact manifold. This allowed them to define a topological index map from the K-theory of the tangent bundle of the foliation to the K-theory of its $\mathrm{C}^{*}$ algebra. Their main result is that the analytic and topological index maps are equal, an extension of the classical Atiyah-Singer families index theorem.

It is well known that the Atiyah-Singer families index theorem follows immediately from the commutative diagram

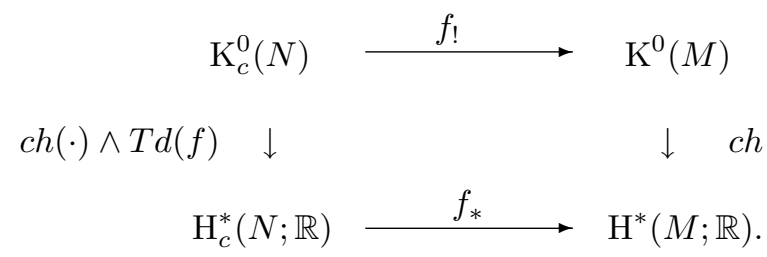

Here $N$ and $M$ are manifolds, with $M$ compact. ch is the usual Chern character, and $f: N \rightarrow M$ is a Koriented map with associated push forward map $f_{!}$. Td $(f)$ is the Todd class of $f$, and $f_{*}$ is the push-forward map in cohomology defined using $f_{*}: \mathrm{H}_{*}(N ; \mathbb{R}) \rightarrow \mathrm{H}_{*}(M ; \mathbb{R})$ and Poincaré Duality.

We extend this result to foliations. Let $F$ be an oriented foliation of $M$ and $f: N \rightarrow M / F$ a K-oriented map to the space of leaves of $F$. Denote by $\mathrm{H}_{c}^{*}(M / F)$ the Haefliger cohomology of $F$. We construct a push forward map in cohomology $f_{*}: \mathrm{H}_{c}^{*}(N ; \mathbb{R}) \rightarrow \mathrm{H}_{c}^{*}(M / F)$. We also construct a Chern character $\operatorname{ch}_{a}: \mathrm{K}_{0}\left(C_{c}^{\infty}(\mathcal{G})\right) \rightarrow \mathrm{H}_{c}^{*}(M / F)$, where $C_{c}^{\infty}(\mathcal{G})$ is the space of smooth functions with compact support on $\mathcal{G}$, the holonomy groupoid of $F$. Denote by $\widehat{F}$ the foliation of $M \times \mathbb{R}^{2 k}$ induced by $F$ with the same leaf dimension. Its holonomy groupoid is $\mathcal{G}^{\mathbb{R}^{2 k}} \simeq \mathcal{G} \times \mathbb{R}^{2 k}$ and its leaf space is $M \times \mathbb{R}^{2 k} / \widehat{F} \simeq M / F \times \mathbb{R}^{2 k}$. Composing $\operatorname{ch}_{a}: \mathrm{K}_{0}\left(C_{0}^{\infty}\left(\mathcal{G}^{\mathbb{R}^{2 k}}\right)\right) \rightarrow \mathrm{H}_{c}^{*}\left(M \times \mathbb{R}^{2 k} / \widehat{F}\right)$ with integration over $\mathbb{R}^{2 k}, \int_{\mathbb{R}^{2 k}}: \mathrm{H}_{c}^{*}\left(M \times \mathbb{R}^{2 k} / \widehat{F}\right) \rightarrow \mathrm{H}_{c}^{*}(M / F)$, gives the map $\operatorname{ch}_{a}^{\mathbb{R}^{2 k}}: \mathrm{K}_{0}\left(C_{c}^{\infty}\left(\mathcal{G}^{\mathbb{R}^{2 k}}\right)\right) \rightarrow \mathrm{H}_{c}^{*}(M / F)$. Given an imbedding of $N$ in $\mathbb{R}^{2 k}$, there is a ConnesSkandalis push forward map $f_{!}^{\mathbb{R}^{2 k}}: \mathrm{K}_{c}^{0}(N) \rightarrow \mathrm{K}_{0}\left(C_{c}^{\infty}\left(\mathcal{G}^{\mathbb{R}^{2 k}}\right)\right)$. We are forced to work with this latter space because in general there is no Bott isomorphism between $\mathrm{K}_{0}\left(C_{c}^{\infty}\left(\mathcal{G}^{\mathbb{R}^{2 k}}\right)\right)$ and $\mathrm{K}_{0}\left(C_{c}^{\infty}(\mathcal{G})\right)$. Our main result is a complete extension of the above result to foliations.

Theorem 5.11. The following diagram commutes,

$$
\begin{aligned}
& \mathrm{K}_{c}^{0}(N) \stackrel{f_{!}^{\mathbb{R}^{2 k}}}{\longrightarrow} \mathrm{K}_{0}\left(C_{c}^{\infty}\left(\mathcal{G}^{\mathbb{R}^{2 k}}\right)\right) \\
& \operatorname{ch}(\cdot) \wedge T d(f) \quad \downarrow \quad \downarrow \quad \operatorname{ch}_{a}^{\mathbb{R}^{2 k}} \\
& \mathrm{H}_{c}^{*}(N ; \mathbb{R}) \longrightarrow f_{*} \longrightarrow \mathrm{H}_{c}^{*}(M / F) .
\end{aligned}
$$


This result extends immediately to other interesting algebras, e.g. the space of superexponentially decaying operators in Connes' $C^{*}$-algebra, [HL02].

Denote by $T F$ the tangent bundle of $F$, and by $\pi: T F \rightarrow M \rightarrow M / F$ the natural map. Then for $k$ sufficiently large, there is a Connes-Skandalis push forward map $\pi_{!}: \mathrm{K}_{c}^{0}(T F) \rightarrow \mathrm{K}_{0}\left(C_{c}^{\infty}\left(\mathcal{G}^{\mathbb{R}^{2 k}}\right)\right)$. By standard methods, Theorem 5.11 immediately yields a cohomological index theorem, namely

Theorem 6.2. For any $u \in \mathrm{K}_{c}^{0}(T F), \operatorname{ch}_{a}^{\mathbb{R}^{2 k}} \circ \pi_{!}(u)$, the algebraic Chern character of the topological index of $u$, is given by

$$
\operatorname{ch}_{a}^{\mathbb{R}^{2 k}} \circ \pi_{!}(u)=(-1)^{p} \int_{F} \pi_{F !}(\operatorname{ch}(u)) \operatorname{Td}(T F \otimes \mathbb{C}) \quad \in \mathrm{H}_{c}^{*}(M / F)
$$

where $\int_{F}: \mathrm{H}^{*}(M ; \mathbb{R}) \rightarrow \mathrm{H}_{c}^{*}(M / F)$ is integration over the leaves of $F, \pi_{F !}: H_{c}^{*}(T F ; \mathbb{R}) \rightarrow \mathrm{H}^{*}(M ; \mathbb{R})$ is integration along the fibers, and Td is the Todd class.

Finally, in [Con86], A. Connes showed that the pairing of any holonomy invariant current extends to the $K$-theory of the $C^{*}$-algebra of the foliation. Therefore, our theorem 6.2 proves, using the ConnesSkandalis index theorem in $K$-theory [CS84] a cohomological index formula in Haefliger cohomology. In particular, our results partially answer a conjecture stated in [He95], namely that the index formula in Haefliger cohomology, for all elliptic pseudodifferential operators on foliations, compare [He95, HL99], is a consequence of the Connes-Skandalis index theorem.

Note that a similar index formula was obtained independently, for Dirac operators on foliations with Hausdorff graph, by Gorokhovsky-Lott, [GL03, GL05].

Acknowledgments. We are indebted to J-M. Bismut, A. Connes, M. Crainic, T. Fack, J. Lott, I. Moerdijk, V. Nistor, H. Oyono-Oyono and G. Skandalis for many useful discussions.

Part of this work was done while the first author was visiting the University of Illinois at Chicago, and while the second author was visiting the Erwin Schrödinger Institute in Vienna, and the University of Lyon I. Both authors are most grateful for the warm hospitality and generous support of their hosts.

\section{Notation And Review}

Throughout the paper, $M$ denotes a smooth compact Riemannian manifold of dimension $n$, and $F$ denotes an oriented foliation of $M$ of dimension $p$ and codimension $q$. So $n=p+q$. The tangent and cotangent bundles of $F$ will be denoted $T F$ and $T^{*} F$ respectively. If $E \rightarrow N$ is a vector bundle over a manifold $N$, we denote the space of smooth sections by $C^{\infty}(E)$ or by $C^{\infty}(N ; E)$ if we want to emphasize the base space of the bundle. The compactly supported sections are denoted by $C_{c}^{\infty}(E)$ or $C_{c}^{\infty}(N ; E)$. The space of differential $k$-forms on $N$ is denoted $\mathcal{A}^{k}(N)$, and we set $\mathcal{A}(N)=\oplus_{k \geq 0} \mathcal{A}^{k}(N)$. The space of compactly supported $k$-forms is denoted $\mathcal{A}_{c}^{k}(N)$, and $\mathcal{A}_{c}(N)=\oplus_{k \geq 0} \mathcal{A}_{c}^{k}(N)$. The tangent and cotangent bundles of $N$ are denoted $T N$ and $T^{*} N$ respectively.

The holonomy groupoid $\mathcal{G}$ of $F$ consists of equivalence classes of paths $\gamma:[0,1] \rightarrow M$ such that the image of $\gamma$ is contained in a leaf of $F$. Two such paths $\gamma_{1}$ and $\gamma_{2}$ are equivalent if $\gamma_{1}(0)=\gamma_{2}(0), \gamma_{1}(1)=\gamma_{2}(1)$, and the holonomy germ along them is the same. Two classes may be composed if one ends where the second begins and the composition is just the juxtaposition of the two paths. This makes $\mathcal{G}$ a groupoid. The space $\mathcal{G}^{(0)}$ of units of $\mathcal{G}$ consists of the equivalence classes of the constant paths, and we identify $\mathcal{G}^{(0)}$ with $M$.

$\mathcal{G}$ is a (in general non-Hausdorff) dimension $2 p+q$ manifold. The basic open sets defining its manifold structure are given as follows. Let $\mathcal{U}$ be a finite good cover of $M$ by foliation charts, [HL90]. Given $U$ and $V$ in this cover and a leafwise path $\gamma$ starting in $U$ and ending in $V$, define $(U, \gamma, V)$ to be the set of equivalence classes of leafwise paths starting in $U$ and ending in $V$ which are homotopic to $\gamma$ through a homotopy of leafwise paths whose end points remain in $U$ and $V$ respectively. It is easy to see, using the holonomy defined by $\gamma$ from a transversal in $U$ to a transversal in $V$, that if $U, V \simeq \mathbb{R}^{p} \times \mathbb{R}^{q}$, then we have the local diffeomorphism $(U, \gamma, V) \simeq \mathbb{R}^{p} \times \mathbb{R}^{p} \times \mathbb{R}^{q}$.

If $\mathcal{G}$ is non-Hausdorff, it is not true that compact sets are always closed, nor that the closure of a compact set is compact. Because of this, we define the notion of having compact support as follows. Given a bundle $E$ over $\mathcal{G}$ and any set $(U, \gamma, V)$ as above, consider $E \mid(U, \gamma, V)$, the restriction of $E$ to $(U, \gamma, V)$. 
The space $C_{c}^{\infty}(E \mid(U, \gamma, V))$ has a natural inclusion into the space of sections of $E$ over $\mathcal{G}$ by extending any element of $C_{c}^{\infty}(E \mid(U, \gamma, V))$ to all of $\mathcal{G}$ by defining it to be zero outside $(U, \gamma, V)$. We define the space $C_{c}^{\infty}(E)=C_{c}^{\infty}(\mathcal{G} ; E)$ of smooth sections of $E$ over $\mathcal{G}$ with compact support to be all finite sums $\sum s_{i}$ where each $s_{i}$ is the image of an element $\widehat{s_{i}}$ in some $C_{c}^{\infty}(E \mid(U, \gamma, V))$. The space of smooth functions with compact support, namely $C_{c}^{\infty}(\mathcal{G} ; \mathcal{G} \times \mathbb{R})$, will be denoted $C_{c}^{\infty}(\mathcal{G})$.

The source and range maps of the groupoid $\mathcal{G}$ are the two natural maps $s, r: \mathcal{G} \rightarrow M$ given by $s([\gamma])=$ $\gamma(0), r([\gamma])=\gamma(1)$. $\mathcal{G}$ has two natural transverse foliations $F_{s}$ and $F_{r}$ whose leaves are respectively $\widetilde{L}_{x}=$ $s^{-1}(x), \widetilde{L}^{x}=r^{-1}(x)$ for $x \in M$. Note that $r: \widetilde{L}_{x} \rightarrow L$ is the holonomy covering of $L$.

The Haefliger cohomology of $F$, [H80], is given as follows. For each $U_{i} \in \mathcal{U}$, let $T_{i} \subset U_{i}$ be a transversal and set $T=\bigcup T_{i}$. We may assume that the closures of the $T_{i}$ are disjoint. Let $\mathcal{H}$ be the holonomy pseudogroup induced by $F$ on $T$. Denote by $\mathcal{A}_{c}^{k}(M / F)$ the quotient of $\mathcal{A}_{c}^{k}(T)$ by the vector subspace generated by elements of the form $\alpha-h^{*} \alpha$ where $h \in \mathcal{H}$ and $\alpha \in \mathcal{A}_{c}^{k}(T)$ has support contained in the range of $h$. Give $\mathcal{A}_{c}^{k}(M / F)$ the quotient topology of the usual $C^{\infty}$ topology on $\mathcal{A}_{c}^{k}(T)$, so this is not a Hausdorff space in general. The exterior derivative $d_{T}: \mathcal{A}_{c}^{k}(T) \rightarrow \mathcal{A}_{c}^{k+1}(T)$ induces a continuous differential $d_{H}: \mathcal{A}_{c}^{k}(M / F) \rightarrow \mathcal{A}_{c}^{k+1}(M / F)$. Note that $\mathcal{A}_{c}^{k}(M / F)$ and $d_{H}$ are independent of the choice of cover $\mathcal{U}$. The complex $\left\{\mathcal{A}_{c}(M / F), d_{H}\right\}$ and its cohomology $\mathrm{H}_{c}^{*}(M / F)$ are, respectively, the Haefliger forms and Haefliger cohomology of $F$.

As the bundle $T F$ is oriented, there is a continuous open surjective linear map, called integration over the leaves,

$$
\int_{F}: \mathcal{A}^{p+k}(M) \longrightarrow \mathcal{A}_{c}^{k}(M / F)
$$

which commutes with the exterior derivatives $d_{M}$ and $d_{H}$. Given $\omega \in \mathcal{A}(M)$, write $\omega=\sum_{i} \omega_{i}$ where $\omega_{i} \in \mathcal{A}_{c}\left(U_{i}\right)$. Integrate $\omega_{i}$ along the fibers of the submersion $\pi_{i}: U_{i} \rightarrow T_{i}$ to obtain $\int_{U_{i}} \omega_{i} \in \mathcal{A}_{c}\left(T_{i}\right)$. Define $\int_{F} \omega \in \mathcal{A}_{c}(M / F)$ to be the class of $\sum_{i} \int_{U_{i}} \omega_{i}$. It is independent of the choice of the $\omega_{i}$ and of the cover $\mathcal{U}$. As $\int_{F}$ commutes with $d_{M}$ and $d_{H}$, it induces the map $\int_{F}: \mathrm{H}^{p+k}(M) \rightarrow \mathrm{H}_{c}^{k}(M / F)$.

\section{TRACES}

In this section, we give some algebraic background and construct the trace which will be used in the sequel.

There is a canonical lift of the normal bundle $\nu$ of $F$ to a bundle $\nu_{\mathcal{G}} \subset T \mathcal{G}$ so that $T \mathcal{G}=T F_{s} \oplus T F_{r} \oplus \nu_{\mathcal{G}}$ and $r_{*} \nu_{\mathcal{G}}=\nu, s_{*} \nu_{\mathcal{G}}=\nu$. It is given as follows. Let $[\gamma] \in \mathcal{G}$ with $s([\gamma])=x, r([\gamma])=y$. Denote by $\exp : \nu \rightarrow M$ the exponential map. Given $X \in \nu_{x}$ and $t \in \mathbb{R}$ sufficiently small, there is a unique curve $\gamma_{t}:[0,1] \rightarrow M$ so that

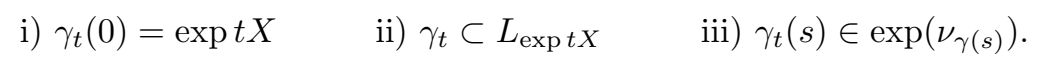

In particular $\gamma_{0}=\gamma$. Thus the family $\left[\gamma_{t}\right]$ in $\mathcal{G}$ defines a tangent vector $\widehat{X} \in T \mathcal{G}_{[\gamma]}$. It is easy to check that $s_{*}(\widehat{X})=X$ and $r_{*}(\widehat{X})$ is the parallel translate of $X$ along $\gamma$ to $\nu_{y}$.

The metric $g_{0}$ on $M$ induces a canonical metric $g_{0}$ on $\mathcal{G}$ as follows. $T \mathcal{G}=T F_{s} \oplus T F_{r} \oplus \nu_{\mathcal{G}}$ and these bundles are mutually orthogonal. On $T F_{r} \oplus \nu_{\mathcal{G}}$ we define $g_{0}$ to be $s^{*} g_{0}$ and on $T F_{s}$ we define $g_{0}$ to be $r^{*}\left(g_{0} \mid T F\right)$.

The foliation $F_{s}$ has normal bundle $\nu_{s}=T F_{r} \oplus \nu_{\mathcal{G}}$, so $\nu_{s} \simeq s^{*}(T M)$ and $\nu_{s}^{*} \simeq s^{*}\left(T^{*} M\right)$. Denote the space of smooth sections of the bundle $\Lambda^{k} T^{*} F_{s} \otimes \Lambda^{h} \nu_{s}^{*}$ by $\mathcal{A}^{k, h}\left(\mathcal{G}, F_{s}\right)$. The exterior derivative $d$ splits with respect to the decomposition

$$
\mathcal{A}^{m}(\mathcal{G}) \simeq \bigoplus_{k+h=m} \mathcal{A}^{k, h}\left(\mathcal{G}, F_{s}\right)
$$

into three bihomogeneous components

$$
d=d_{F}+d_{\nu}+d_{-1,2},
$$


where $d_{F}$ is the leafwise differential for $F_{s}$ with bidegree $(1,0)$ with respect to the splitting but which is independent of the choice of $\nu$ [BN03], $d_{\nu}$ is the $(0,1)$ homogeneous component, and $d_{-1,2}$ is an extra differential which has bidegree $(-1,2)$, see [T97] for more details.

Let $E_{1}$ be a complex vector bundle over $M$ with Hermitian metric and connection, and set $E=r^{*} E_{1}$ with the pulled back metric and connection, denoted $\nabla$. We may regard $\nabla$ as an operator of degree one on $C^{\infty}\left(E \otimes \Lambda T^{*} \mathcal{G}\right)$ where on decomposable sections $\phi \otimes \omega, \nabla(\phi \otimes \omega)=(\nabla \phi) \wedge \omega+\phi \otimes d \omega$. Then $\nabla$ defines a quasi-connection $\nabla^{\nu}$ acting on $C^{\infty}\left(E \otimes \Lambda \nu_{s}^{*}\right)$ by the composition

$$
C^{\infty}\left(E \otimes \Lambda \nu_{s}^{*}\right) \stackrel{i}{\longrightarrow} C^{\infty}\left(E \otimes \Lambda T^{*} \mathcal{G}\right) \stackrel{\nabla}{\longrightarrow} C^{\infty}\left(E \otimes \Lambda T^{*} \mathcal{G}\right) \stackrel{p_{\nu}}{\longrightarrow} C^{\infty}\left(E \otimes \Lambda \nu_{s}^{*}\right),
$$

where $i$ is the inclusion and $p_{\nu}$ is induced by the projection $p_{\nu}: T^{*} \mathcal{G} \rightarrow \nu_{s}^{*}$. Given $\omega \in C^{\infty}\left(\Lambda \nu_{s}^{*}\right)$, denote by $M_{\omega}: C^{\infty}\left(E \otimes \Lambda \nu_{s}^{*}\right) \rightarrow C^{\infty}\left(E \otimes \Lambda \nu_{s}^{*}\right)$ the operator given by exterior multiplication on the left by $\omega$. Then for any $\omega \in C^{\infty}\left(\Lambda^{j} \nu_{s}^{*}\right)$ and any $\phi \in C^{\infty}\left(E \otimes \Lambda \nu_{s}^{*}\right), \nabla^{\nu}$ satisfies the relation

$$
\nabla^{\nu}(\omega \phi)-(-1)^{j} \omega \nabla^{\nu} \phi=\left(d_{\nu} \omega\right) \phi
$$

In other words, the graded commutator $\left[\nabla^{\nu}, M_{\omega}\right]$ of these operators on $C^{\infty}\left(E \otimes \Lambda \nu_{s}^{*}\right)$ is the operator $M_{d_{\nu} \omega}$ of exterior multiplication (on the left) by $d_{\nu} \omega$.

If $T \in \operatorname{End}\left(C^{\infty}\left(E \otimes \Lambda \nu_{s}^{*}\right)\right)$ we say that $T$ is homogeneous of degree $k$ if it maps $E \otimes \Lambda^{j} \nu_{s}^{*}$ to $E \otimes \Lambda^{j+k} \nu_{s}^{*}$. $C^{\infty}\left(E \otimes \Lambda \nu_{s}^{*}\right)$ is an $\mathcal{A}(M)$-module where for $\phi \in C^{\infty}\left(E \otimes \Lambda \nu_{s}^{*}\right)$, and $\omega \in \mathcal{A}(M)$, we set

$$
M_{\omega} \phi=p_{\nu}\left(s^{*}(\omega)\right) \phi
$$

where again the map $p_{\nu}: \mathcal{A}(\mathcal{G}) \rightarrow C^{\infty}\left(\Lambda \nu_{s}^{*}\right)$ is induced by the projection $p_{\nu}: T^{*} \mathcal{G} \rightarrow \nu_{s}^{*}$. Denote by $\Omega^{k}$ the space of $\mathcal{A}(M)$-equivariant endomorphisms of $C^{\infty}\left(E \otimes \Lambda \nu_{s}^{*}\right)$ with degree $k$ and set

$$
\Omega=\bigoplus_{k} \Omega^{k}
$$

Then $T \in \Omega^{k}$ if and only if for all $\omega \in \mathcal{A}^{j}(M)$,

$$
\left[T, M_{\omega}\right] \equiv T \circ M_{\omega}-(-1)^{k j} M_{\omega} \circ T=0 .
$$

A pseudo-differential $\mathcal{G}$-operator with uniform support acting on $E$ is a smooth family $\left(P_{x}\right)_{x \in M}$ of $\mathcal{G}$ invariant pseudo-differential operators, where for each $x, P_{x}$ is an operator acting on $E \mid \widetilde{L}_{x}$. The $\mathcal{G}$-invariance property means that for any $\gamma \in \widetilde{L}_{x}^{y}=\widetilde{L}_{x} \cap \widetilde{L}^{y}$, we have

$$
(\gamma \cdot P)_{y} \equiv U_{\gamma} \circ P_{x} \circ U_{\gamma}^{-1}=P_{y},
$$

where $U_{\gamma}: C_{c}^{\infty}\left(\widetilde{L}_{x}, E\right) \rightarrow C_{c}^{\infty}\left(\widetilde{L}_{y}, E\right)$ is the induced action of the groupoid $\mathcal{G}$ defined by

$$
\left[U_{\gamma} \xi\right]\left(\gamma^{\prime}\right)=\xi\left(\gamma^{\prime} \gamma\right), \quad \gamma^{\prime} \in \widetilde{L}_{y}
$$

The smoothness assumption is rigorously defined in [NWX96]. If we denote by $K_{x}$ the Schwartz kernel of $P_{x}$, then the $\mathcal{G}$-invariance assumption implies that the family $\left(K_{x}\right)_{x \in M}$ induces a distributional section $K$ of the bundle $\operatorname{Hom}(E)$ over the holonomy groupoid, whose fiber at a point $\gamma$ is a homomorphism from $E_{s(\gamma)}$ to $E_{r(\gamma)}$, which is smooth outside $\mathcal{G}^{(0)}=M$. Since $M$ is compact, the uniform support condition becomes the assumption that the support of $K$ is compact in $\mathcal{G}$. We denote by $\Psi^{m}(\mathcal{G} ; E)$ the space of all such uniformly supported pseudo-differential $\mathcal{G}$-operators of order $m$, i.e. such that each $P_{x}$ has order $m$. $\Psi^{m}(\mathcal{G} ; E)$ is a $C^{\infty}(M)$ module, where $f \in C^{\infty}(M)$ acts by multiplication by $s^{*}(f)$. The space of all uniformly supported pseudo-differential $\mathcal{G}$-operators is denoted $\Psi^{\infty}(\mathcal{G} ; E)$ and the space of uniformly supported regularizing $\mathcal{G}$ operators is denoted $\Psi^{-\infty}(\mathcal{G} ; E)$. So,

$$
\Psi^{\infty}(\mathcal{G} ; E)=\bigcup_{m \in \mathbb{Z}} \Psi^{m}(\mathcal{G} ; E) \text { and } \Psi^{-\infty}(\mathcal{G} ; E)=\bigcap_{m \in \mathbb{Z}} \Psi^{m}(\mathcal{G} ; E) .
$$

The Schwartz Kernel Theorem identifies $\Psi^{-\infty}(\mathcal{G} ; E)$ with $C_{c}^{\infty}(\mathcal{G}, \operatorname{Hom}(E))$, see [NWX96]. We may consider the algebra

$$
\Psi^{-\infty}(\mathcal{G} ; E) \widehat{\otimes}_{C^{\infty}(M)} \mathcal{A}(M)
$$

as a subspace of $\Omega$ by using the $\mathcal{A}(M)$ module structure of $C^{\infty}\left(E \otimes \wedge \nu_{s}^{*}\right)$. 
Denote by $\partial_{\nu}: \operatorname{End}\left(C^{\infty}\left(E \otimes \Lambda \nu_{s}^{*}\right)\right) \rightarrow \operatorname{End}\left(C^{\infty}\left(E \otimes \Lambda \nu_{s}^{*}\right)\right)$ the linear operator which on homogeneous elements of degree $k$ is given by

$$
\partial_{\nu}(T)=\left[\nabla^{\nu}, T\right]=\nabla^{\nu} \circ T-(-1)^{k} T \circ \nabla^{\nu} .
$$

Lemma 3.1. $\partial_{\nu}$ preserves the subspace $\Omega$, and $\left(\partial_{\nu}\right)^{2}$ is given by the commutator with $\theta=\left(\nabla^{\nu}\right)^{2} \in \Omega^{2}$. In addition, $\partial_{\nu}$ preserves the subspace $\Psi^{-\infty}(\mathcal{G} ; E) \widehat{\otimes}_{C^{\infty}(M)} \mathcal{A}(M)$.

Proof. Let $T$ be a homogeneous endomorphism of $C^{\infty}\left(E \otimes \Lambda \nu_{s}^{*}\right)$ of degree $k$ and let $\omega \in \mathcal{A}^{j}(M)$. Then

$$
\begin{aligned}
{\left[\partial_{\nu}(T), M_{\omega}\right]=} & \nabla^{\nu} \circ T \circ M_{\omega}-(-1)^{k} T \circ \nabla^{\nu} \circ M_{\omega}- \\
& (-1)^{(k+1) j} M_{\omega} \circ \nabla^{\nu} \circ T+(-1)^{(k+1) j}(-1)^{k} M_{\omega} \circ T \circ \nabla^{\nu} \\
= & (-1)^{k j}\left(\nabla^{\nu} \circ M_{\omega} \circ T-(-1)^{k} T \circ\left((-1)^{j} M_{\omega} \circ \nabla^{\nu}+M_{d_{\nu} \omega}\right)-\right. \\
& (-1)^{k j+j} M_{\omega} \circ \nabla^{\nu} \circ T+(-1)^{k j+j+k} M_{\omega} \circ T \circ \nabla^{\nu} \\
= & (-1)^{k j+j} M_{\omega} \circ \nabla^{\nu} \circ T+(-1)^{k j} M_{d_{\nu} \omega} \circ T-(-1)^{k+j+k j} M_{\omega} \circ T \circ \nabla^{\nu}- \\
& (-1)^{k(j+1)+k} M_{d_{\nu} \omega} \circ T-(-1)^{k j+j} M_{\omega} \circ \nabla^{\nu} \circ T+(-1)^{k j+k+j} M_{\omega} \circ T \circ \nabla^{\nu} \\
= & 0 .
\end{aligned}
$$

In the same way we have

$$
\begin{aligned}
\left(\partial_{\nu} \circ \partial_{\nu}\right)(T) & =\nabla^{\nu} \circ \partial_{\nu}(T)-(-1)^{k+1} \partial_{\nu}(T) \circ \nabla^{\nu} \\
& =\nabla^{\nu} \circ\left(\nabla^{\nu} \circ T-(-1)^{k} T \circ \nabla^{\nu}\right)-(-1)^{k+1}\left(\nabla^{\nu} \circ T-(-1)^{k} T \circ \nabla^{\nu}\right) \circ \nabla^{\nu} \\
& =[\theta, T] .
\end{aligned}
$$

Finally, in order to prove that $\partial_{\nu}$ preserves $\Psi^{-\infty}(\mathcal{G} ; E) \widehat{\otimes}_{C^{\infty}(M)} \mathcal{A}(M)$, we use the $\mathcal{A}(M)$-module structure to reduce to the case where the element $T$ under consideration actually belongs to $\Psi^{-\infty}(\mathcal{G} ; E)=$ $C_{c}^{\infty}(\mathcal{G} ; \operatorname{Hom}(E))$. By linearity, we may assume that $T$ comes from a compactly supported smooth Schwartz kernel $K \in C_{c}^{\infty}((U, \gamma, V) ; \operatorname{Hom}(E))$ for some basic open set $(U, \gamma, V)$. But then it is obvious that $\partial_{\nu}(K)$ is also smooth. Thus the proof is complete.

For $T \in \Psi^{-\infty}(\mathcal{G} ; E) \widehat{\otimes}_{C^{\infty}(M)} \mathcal{A}^{k}(M)=C_{c}^{\infty}(\mathcal{G} ; \operatorname{Hom}(E)) \widehat{\otimes}_{C^{\infty}(M)} \mathcal{A}^{k}(M)$, define the trace of $T$ to be the Haefliger $k$-form $\operatorname{Tr}(T)$ given by

$$
\operatorname{Tr}(T)=\int_{F} \operatorname{tr}(K(\bar{x}, \bar{x})) d x
$$

where $K$ is the smooth Schwartz kernel of $T, \bar{x}$ is the class of the constant path at $x, \operatorname{tr}(K(\bar{x}, \bar{x}))$ is the usual trace of $K(\bar{x}, \bar{x}) \in \operatorname{End}\left(E_{\bar{x}}\right) \otimes \Lambda^{k} T^{*} M_{x}$ and so belongs to $\Lambda^{k} T^{*} M_{x}$, and $d x$ is the leafwise volume form associated with the fixed orientation of the foliation $F$.

Lemma 3.2. The map $\operatorname{Tr}: \Psi^{-\infty}(\mathcal{G} ; E) \widehat{\otimes}_{C^{\infty}(M)} \mathcal{A}(M) \rightarrow \mathcal{A}_{c}(M / F)$ is a graded trace which satisfies $\operatorname{Tr} \circ \partial_{\nu}=$ $d_{H} \circ \operatorname{Tr}$.

Proof. This is a corollary of Lemma 2.5, p. 443 of [HL02] which says that if $K$ is a smooth section of $\operatorname{Hom}(E) \widehat{\otimes}_{C^{\infty}(M)} \mathcal{A}(M)$ over $\mathcal{G}$ which is uniformly exponentially decaying (considered as a family of sections over the $\left.\widetilde{L}_{x} \times \widetilde{L}_{x}\right)$ and $H$ is a continuous section of $\operatorname{Hom}(E) \widehat{\otimes}_{C \infty(M)} \mathcal{A}(M)$ over $\mathcal{G}$ with uniformly bounded coefficients, then $\operatorname{Tr}([H, K])=0$. As the the kernels in question here are all uniformly supported, they always have uniformly bounded coefficients. Likewise, for any such kernel, there is an $R>0$ so that on any $\widetilde{L}_{x} \times \widetilde{L}_{x}$, the kernel is zero off the $R$ neighborhood of the diagonal and so is uniformly exponentially decaying.

Now suppose that $T \in \Psi^{-\infty}(\mathcal{G} ; E) \widehat{\otimes}_{C \infty(M)} \mathcal{A}(M)$. As $\nabla^{\nu}$ is local operator and everything is linear, we may assume that $T$ is given by a section $K \in C_{c}^{\infty}\left((U, \bar{w}, U) ; \operatorname{Hom}(E) \otimes \Lambda T^{*} U\right)$, where $w \in U$. The foliation chart $U \simeq \mathbb{R}^{p} \times \mathbb{R}^{q}$, and $(U, \bar{w}, U) \simeq \mathbb{R}^{p} \times \mathbb{R}^{q} \times \mathbb{R}^{p}$, with coordinates $(x, y, z)$. The map $s:(U, \bar{w}, U) \rightarrow U$ is 
given by $s(x, y, z)=(x, y)$ and the foliation $F_{s}$ restricted to $(U, \bar{w}, U)$ has leaves of the form $(x, y) \times \mathbb{R}^{p}$. In this setting, $\nabla^{\nu}=d_{x}+d_{y}+A$ where $A \in C^{\infty}((U, \bar{w}, U) ; \operatorname{Hom}(E)) \widehat{\otimes}_{C^{\infty}(U)} \mathcal{A}^{1}(U)$. Thus

$$
\operatorname{Tr}\left(\partial_{\nu} T\right)=\int_{F} \operatorname{tr}\left(\left[d_{x}+d_{y}+A, K\right] d x=\int_{F} \operatorname{tr}\left(d_{x} K\right) d x+\int_{F} \operatorname{tr}\left(d_{y} K\right) d x+\int_{F} \operatorname{tr}([A, K]) d x .\right.
$$

The term $\int_{F} \operatorname{tr}\left(d_{x} K\right) d x$ is obviously zero. A direct computation shows that $\int_{F} \operatorname{tr}([A, K]) d x=0$. Finally, note that in these coordinates, $d_{H}$ is $d_{y}$ if we take the transversal in $U$ to be $(0,0) \times \mathbb{R}^{q}$. Thus $\int_{F} \operatorname{tr}\left(d_{y} K\right) d x=$ $\int_{F} d_{y} \operatorname{tr}(K) d x=d_{H} \int_{F} \operatorname{tr}(K) d x=d_{H} \operatorname{Tr}(T)$.

Since $\partial_{\nu}^{2}$ is not necessarily zero, we now employ Connes' $X$-trick to construct a new graded differential algebra $\left(\widetilde{\mathcal{A}}_{-\infty}, \delta\right)$ out of the graded quasi-differential algebra $\left(\mathcal{A}_{c}^{\infty}\left(\mathcal{G} \mid F_{s} ; E\right), \partial_{\nu}\right)$, see [Con94], p. 229. Write $\mathcal{A}_{-\infty}$ for $\mathcal{A}_{c}^{\infty}\left(\mathcal{G} \mid F_{s} ; E\right)$ and note that the curvature operator $\theta=\nabla^{\nu} \circ \nabla^{\nu}$ is a multiplier of $\mathcal{A}_{-\infty}$. As a vector space $\widetilde{\mathcal{A}}_{-\infty}=M_{2}\left(\mathcal{A}_{-\infty}\right)$. An element $\tilde{T} \in \widetilde{\mathcal{A}}_{-\infty}$ is homogeneous of degree $\partial \tilde{T}=k$ if

$$
k=\partial \tilde{T}_{11}=\partial \tilde{T}_{12}+1=\partial \tilde{T}_{21}+1=\partial \tilde{T}_{22}+2
$$

The differential $\delta$ on $\widetilde{\mathcal{A}}_{-\infty}$ is defined on elements of the form $\left(\begin{array}{cc}\tilde{T}_{11} & 0 \\ 0 & 0\end{array}\right)$ by

$$
\delta \tilde{T}=\left(\begin{array}{cc}
\partial_{\nu} \tilde{T}_{11} & (-1)^{\partial T_{11}} \tilde{T}_{11} \\
\tilde{T}_{11} & 0
\end{array}\right)
$$

for homogeneous $\tilde{T}_{11} \in \mathcal{A}_{-\infty}$. On homogeneous elements of $\tilde{\mathcal{A}}_{-\infty}$ it is given by

$$
\delta \tilde{T}=\left(\begin{array}{cc}
\partial_{\nu} \tilde{T}_{11} & \partial_{\nu} \tilde{T}_{12} \\
-\partial_{\nu} \tilde{T}_{21} & -\partial_{\nu} \tilde{T}_{22}
\end{array}\right)+\left(\begin{array}{cc}
0 & -\theta \\
1 & 0
\end{array}\right) \tilde{T}+(-1)^{\partial \tilde{T}} \tilde{T}\left(\begin{array}{cc}
0 & 1 \\
-\theta & 0
\end{array}\right) .
$$

A straightforward computation gives $\delta^{2}=0$. Set

$$
\Theta=\left(\begin{array}{ll}
1 & 0 \\
0 & \theta
\end{array}\right)
$$

and define a new product on $\widetilde{\mathcal{A}}_{-\infty}$ by

$$
\tilde{T} * \tilde{T}^{\prime}=\tilde{T} \Theta \tilde{T}^{\prime}
$$

This makes $\left(\widetilde{\mathcal{A}}_{-\infty}, \delta\right)$ a graded differential algebra.

The graded algebra $\mathcal{A}_{-\infty}$ embeds as a subalgebra of $\widetilde{\mathcal{A}}_{-\infty}$ by using the map

$$
T \hookrightarrow\left(\begin{array}{cc}
T & 0 \\
0 & 0
\end{array}\right)
$$

We shall therefore also denote by $T$ the image in $\widetilde{\mathcal{A}}_{-\infty}$ of any $T \in \mathcal{A}_{-\infty}$.

For homogeneous $\tilde{T} \in \widetilde{\mathcal{A}}_{-\infty}$ define

$$
\Phi(\tilde{T})=\operatorname{Tr}\left(\tilde{T}_{11}\right)-(-1)^{\partial \tilde{T}} \operatorname{Tr}\left(\tilde{T}_{22} \theta\right),
$$

and extend to arbitrary elements by linearity.

Theorem 3.3. The map $\Phi: \widetilde{\mathcal{A}}_{-\infty} \rightarrow \mathcal{A}_{c}^{*}(M / F)$ is a graded trace, and $\Phi \circ \delta=d_{H} \circ \Phi$.

Proof. Let $\tilde{T}, \tilde{S}$ be two homogeneous elements of $\widetilde{\mathcal{A}}_{-\infty}$ with degree respectively $k$ and $\ell$. Then

$$
\Phi([\tilde{T}, \tilde{S}])=\operatorname{Tr}\left(\left[\tilde{T}_{11}, \tilde{S}_{11}\right]\right)+\operatorname{Tr}\left(\left[\tilde{T}_{12} \theta, \tilde{S}_{21}\right]\right)+(-1)^{k+\ell+1} \operatorname{Tr}\left(\left[\tilde{T}_{21}, \tilde{S}_{12} \theta\right]\right)+(-1)^{k+\ell+1} \operatorname{Tr}\left(\left[\tilde{T}_{22} \theta, \tilde{S}_{22} \theta\right]\right)=0
$$

by Lemma 3.2 .

For a homogeneous element $\tilde{T} \in \widetilde{\mathcal{A}}_{-\infty}^{k-1}$,

$$
(\Phi \circ \delta)(\tilde{T})=\left(\operatorname{Tr} \circ \partial_{\nu}\right)\left(\tilde{T}_{11}\right)+(-1)^{k}\left(\operatorname{Tr} \circ \partial_{\nu}\right)\left(\tilde{T}_{22} \theta\right)-\left(\operatorname{Tr} \circ\left(\partial_{\nu}\right)^{2}\right)\left(\tilde{T}_{21}\right),
$$


since $\partial_{\nu}(\theta)=0$. Using Lemma 3.2 again gives

$$
(\Phi \circ \delta)(\tilde{T})=\left(d_{H} \circ \operatorname{Tr}\right)\left(\tilde{T}_{11}+(-1)^{k} \tilde{T}_{22} \theta\right)-\left(d_{H}^{2} \circ \operatorname{Tr}\right)\left(\tilde{T}_{21}\right) .
$$

Since $d_{H}^{2}=0$, we get

$$
(\Phi \circ \delta)(\tilde{T})=d_{H}\left(\operatorname{Tr} \tilde{T}_{11}+(-1)^{k} \operatorname{Tr} \tilde{T}_{22} \theta\right)
$$

and the proof is complete.

\section{The Algebraic Chern character}

In this section, we use the map $\Phi$ to construct an algebraic Chern-Connes character

$$
\operatorname{ch}_{a}: K_{0}\left(C_{c}^{\infty}(\mathcal{G})\right) \longrightarrow \mathrm{H}_{c}^{*}(M / F) .
$$

As $\mathrm{K}_{0}\left(C_{c}^{\infty}(\mathcal{G})\right) \simeq \mathrm{K}_{0}\left(C_{c}^{\infty}(\mathcal{G} ; \operatorname{Hom}(E))\right) \simeq \mathrm{K}_{0}\left(\Psi^{-\infty}(\mathcal{G} ; E)\right)$, we may work with $\mathrm{K}_{0}\left(\Psi^{-\infty}(\mathcal{G}, E)\right)$ in place of $\mathrm{K}_{0}\left(C_{c}^{\infty}(\mathcal{G})\right)$. Denote by $M_{N}\left(\Psi^{-\infty}(\mathcal{G} ; E) \oplus \mathbb{C}\right)$ the space of $N \times N$ matrices with coefficients in $\Psi^{-\infty}(\mathcal{G} ; E) \oplus \mathbb{C}$.

Theorem 4.1. Let $B=\left[\tilde{e}_{1}\right]-\left[\tilde{e}_{2}\right]$ be an element of $K_{0}\left(\Psi^{-\infty}(\mathcal{G} ; E)\right)$, where $\tilde{e}_{1}=\left(e_{1}, \lambda_{1}\right)$ and $\tilde{e}_{2}=\left(e_{2}, \lambda_{2}\right)$ are idempotents in $M_{N}\left(\Psi^{-\infty}(\mathcal{G} ; E) \oplus \mathbb{C}\right)$, which we consider as elements of $M_{N}\left(\widetilde{\mathcal{A}}_{-\infty} \oplus M_{2}(\mathbb{C})\right)$. Then the Haefliger form

$$
(\Phi \circ \operatorname{tr})\left(e_{1} \exp \left(\frac{-\left(\delta e_{1}\right)^{2}}{2 i \pi}\right)\right)-(\Phi \circ \operatorname{tr})\left(e_{2} \exp \left(\frac{-\left(\delta e_{2}\right)^{2}}{2 i \pi}\right)\right)
$$

is closed and its Haefliger cohomology class depends only on B. Here $\operatorname{tr}: M_{N}\left(\widetilde{\mathcal{A}}_{-\infty}\right) \rightarrow \widetilde{\mathcal{A}}_{-\infty}$ is the usual trace.

Definition 4.2. Let $B=\left[\tilde{e}_{1}\right]-\left[\tilde{e}_{2}\right] \in K_{0}\left(\Psi^{-\infty}(\mathcal{G} ; E)\right)$ where $\tilde{e}_{1}=\left(e_{1}, \lambda_{1}\right)$ and $\tilde{e}_{2}=\left(e_{2}, \lambda_{2}\right)$ are idempotents. The algebraic Chern character $\operatorname{ch}_{a}(B)$ of $B$ is the Haefliger cohomology class

$$
\operatorname{ch}_{a}(B)=\left[(\Phi \circ \operatorname{tr})\left(e_{1} \exp \left(\frac{-\left(\delta e_{1}\right)^{2}}{2 i \pi}\right)\right)\right]-\left[(\Phi \circ \operatorname{tr})\left(e_{2} \exp \left(\frac{-\left(\delta e_{2}\right)^{2}}{2 i \pi}\right)\right)\right] \text {. }
$$

Proof. The derivation $\delta$ extends to a derivation on $\widetilde{\mathcal{A}}_{-\infty} \oplus \mathbb{C}$ (and so also to $\left.M_{N}\left(\widetilde{\mathcal{A}}_{-\infty} \oplus \mathbb{C}\right)\right)$ by setting $\delta(e, \lambda)=(\delta e, 0)$. In addition, $\Phi$ extends to a graded trace $\Phi: \widetilde{\mathcal{A}}_{-\infty} \oplus \mathbb{C} \rightarrow \mathcal{A}_{c}(M / F)$ by setting $\Phi((e, \lambda))=$ $\Phi(e)$, and Theorem 3.3 remains valid for this extended trace.

Let $e \in M_{N}\left(\widetilde{\mathcal{A}}_{-\infty} \oplus M_{2}(\mathbb{C})\right)$ be an idempotent, and set $u=2 e-1$. Then

$$
u^{2}=1 \text { and } u \cdot \delta e+\delta e \cdot u=0 .
$$

By Theorem 3.3, $d_{H}(\Phi \circ \operatorname{tr})\left(e(\delta e)^{2 k}\right)=(\Phi \circ \operatorname{tr})\left((\delta e)^{2 k+1}\right)$ and we have

$$
(\Phi \circ \operatorname{tr})\left((\delta e)^{2 k+1}\right)=(\Phi \circ \operatorname{tr})\left(u^{2}(\delta e)^{2 k+1}\right)=-(\Phi \circ \operatorname{tr})\left(u(\delta e)^{2 k+1} u\right) .
$$

The last equality is a consequence of the relation $u \cdot \delta e=-\delta e \cdot u$. On the other hand, since $\Phi \circ \operatorname{tr}$ is a trace, we have

so

$$
-(\Phi \circ \operatorname{tr})\left(u(\delta e)^{2 k+1} u\right)=-(\Phi \circ \operatorname{tr})\left((\delta e)^{2 k+1} u^{2}\right)=-(\Phi \circ \operatorname{tr})\left((\delta e)^{2 k+1}\right),
$$

$$
d_{H}\left((\Phi \circ \operatorname{tr})\left(e(\delta e)^{2 k}\right)\right)=0 .
$$

Now suppose $e_{t}$ is a smooth path of idempotents in $M_{N}\left(\Psi^{-\infty}(\mathcal{G} ; E) \oplus \mathbb{C}\right)$ and set $a_{t}=\frac{d e_{t}}{d t}\left(2 e_{t}-1\right)$. Then

$$
\begin{aligned}
{\left[a_{t}, e_{t}\right] } & =a_{t} e_{t}-e_{t} a_{t} \\
& =\frac{d e_{t}}{d t}\left(2 e_{t}-1\right) e_{t}-e_{t} \frac{d e_{t}}{d t}\left(2 e_{t}-1\right) \\
& =\frac{d e_{t}}{d t} e_{t}+e_{t} \frac{d e_{t}}{d t}-2 e_{t} \frac{d e_{t}}{d t} e_{t}
\end{aligned}
$$


Since $e_{t}^{2}=e_{t}$ for any $t$, we have

so

$$
\frac{d e_{t}}{d t} e_{t}+e_{t} \frac{d e_{t}}{d t}=\frac{d e_{t}}{d t} \text { and } e_{t} \frac{d e_{t}}{d t} e_{t}=0
$$

$$
\frac{d e_{t}}{d t}=\left[a_{t}, e_{t}\right]
$$

This implies that the zero th order component of $\frac{d}{d t}(\Phi \circ \operatorname{tr})\left(e_{t} \exp \left[\frac{-\left(\delta e_{t}\right)^{2}}{2 i \pi}\right]\right)$ is zero.

For any $k \geq 1$ we have by multilinearity

$$
\begin{gathered}
\frac{d}{d t}(\Phi \circ \operatorname{tr})\left(e_{t}\left(\delta e_{t}\right)^{2 k}\right)=(\Phi \circ \operatorname{tr})\left(\frac{d e_{t}}{d t}\left(\delta e_{t}\right)^{2 k}\right)+\sum_{j=1}^{2 k}(\Phi \circ \operatorname{tr})\left(e_{t}\left(\delta e_{t}\right)^{j-1}\left(\delta \frac{d e_{t}}{d t}\right)\left(\delta e_{t}\right)^{2 k-j}\right) \\
=(\Phi \circ \operatorname{tr})\left(\left[a_{t}, e_{t}\right]\left(\delta e_{t}\right)^{2 k}\right)+\sum_{j=1}^{2 k}(\Phi \circ \operatorname{tr})\left(e_{t}\left(\delta e_{t}\right)^{j-1} \delta\left[a_{t}, e_{t}\right]\left(\delta e_{t}\right)^{2 k-j}\right) \\
=(\Phi \circ \operatorname{tr})\left(\left[a_{t}, e_{t}\right]\left(\delta e_{t}\right)^{2 k}\right)+\sum_{j=1}^{2 k}(\Phi \circ \operatorname{tr})\left(\left[e_{t}\left(\delta e_{t}\right)^{j-1} a_{t}\left(\delta e_{t}\right)^{2 k-j+1}-e_{t}\left(\delta e_{t}\right)^{j} a_{t}\left(\delta e_{t}\right)^{2 k-j}\right]\right)+ \\
\sum_{j=1}^{2 k}(\Phi \circ \operatorname{tr})\left(e_{t}\left(\delta e_{t}\right)^{j-1}\left[\left(\delta a_{t}\right) e_{t}-e_{t}\left(\delta a_{t}\right)\right]\left(\delta e_{t}\right)^{2 k-j}\right) .
\end{gathered}
$$

The first sum collapses to

$$
(\Phi \circ \operatorname{tr})\left(e_{t} a_{t}\left(\delta e_{t}\right)^{2 k}\right)-(\Phi \circ \operatorname{tr})\left(e_{t}\left(\delta e_{t}\right)^{2 k} a_{t}\right)=-(\Phi \circ \operatorname{tr})\left(\left[a_{t}, e_{t}\right]\left(\delta e_{t}\right)^{2 k}\right),
$$

so the first two terms cancel.

To analyze the second sum, note that since $e_{t}$ is an idempotent and $\delta$ is a derivation, by induction we have

$$
\begin{gathered}
e_{t}\left(\delta e_{t}\right)^{2 h} e_{t}=e_{t}\left(\delta e_{t}\right)^{2 h}=\left(\delta e_{t}\right)^{2 h} e_{t}, \quad e_{t}\left(\delta e_{t}\right)^{2 h+1} e_{t}=0, \\
\text { and } \quad e_{t}\left(\delta e_{t}\right)^{2 h+1}+\left(\delta e_{t}\right)^{2 h+1} e_{t}=\left(\delta e_{t}\right)^{2 h+1} \quad \forall h \geq 0 .
\end{gathered}
$$

The second sum is then

$$
\sum_{j=1}^{2 k}(\Phi \circ \operatorname{tr})\left(e_{t}\left(\delta e_{t}\right)^{j-1}\left(\delta a_{t}\right) e_{t}\left(\delta e_{t}\right)^{2 k-j}-e_{t}\left(\delta e_{t}\right)^{j-1} e_{t}\left(\delta a_{t}\right)\left(\delta e_{t}\right)^{2 k-j}\right) .
$$

Using the fact that $\Phi \circ$ tr is a graded trace, this equals

$$
\begin{gathered}
\sum_{j=1}^{2 k}(\Phi \circ \operatorname{tr})\left((-1)^{j} e_{t}\left(\delta e_{t}\right)^{2 k-j} e_{t}\left(\delta e_{t}\right)^{j-1}\left(\delta a_{t}\right)\right)+\sum_{j=1}^{2 k}(\Phi \circ \operatorname{tr})\left((-1)^{(j+1)}\left(\delta e_{t}\right)^{2 k-j} e_{t}\left(\delta e_{t}\right)^{j-1} e_{t}\left(\delta a_{t}\right)\right) \\
=\sum_{i=1}^{k}(\Phi \circ \operatorname{tr})\left(e_{t}\left(\delta e_{t}\right)^{2 k-2 i} e_{t}\left(\delta e_{t}\right)^{2 i-1}\left(\delta a_{t}\right)\right)+\sum_{i=1}^{k}(\Phi \circ \operatorname{tr})\left(\left(\delta e_{t}\right)^{2 k-2 i+1} e_{t}\left(\delta e_{t}\right)^{2 i-2} e_{t}\left(\delta a_{t}\right)\right) \\
=\sum_{i=1}^{k}(\Phi \circ \operatorname{tr})\left(e_{t}\left(\delta e_{t}\right)^{2 k-1}\left(\delta a_{t}\right)\right)+\sum_{i=1}^{k}(\Phi \circ \operatorname{tr})\left(-e_{t}\left(\delta e_{t}\right)^{2 k-1}\left(\delta a_{t}\right)+\left(\delta e_{t}\right)^{2 k-1}\left(\delta a_{t}\right)\right) \\
=\sum_{i=1}^{k}(\Phi \circ \operatorname{tr})\left(\left(\delta e_{t}\right)^{2 k-1}\left(\delta a_{t}\right)\right)=k d_{H}(\Phi \circ \operatorname{tr})\left(e_{t}\left(\delta e_{t}\right)^{2 k-2}\left(\delta a_{t}\right)\right) .
\end{gathered}
$$

Thus $\operatorname{ch}_{a}(B)$ does not depend on the choice of representative of $B$.

Now suppose that ${ }^{t} \nabla$ is a smooth family of connections on $E_{1}$ with associated differentials $\partial_{t}$, and $\delta_{t}$. On $M \times I$, where $I=[0,1]$, we have the foliation $\widehat{F}$ whose leaves are of the form $L \times\{t\}, t \in I$. Then 
$\mathcal{G}_{\widehat{F}}=\mathcal{G} \times I$, and $M \times I / \widehat{F}=M / F \times I$. Denote by $\pi: \mathcal{G} \times I \rightarrow \mathcal{G}$ the projection. On the bundle $\pi^{*} E$, we have the connection $\nabla$ determined by ${ }^{t} \nabla$. In particular, for $\phi \in C^{\infty}(E)$, and $X \in T(\mathcal{G} \times I)$, $\nabla_{X}(\phi \circ \pi)(x, t) \equiv{ }^{t} \nabla_{\pi_{*}(X)}(\phi)(x)$, where we identify $\pi^{*} E_{(x, t)}$ with $E_{x}$. Thus on $\mathcal{G} \times\{t\}, \nabla={ }^{t} \nabla+d_{t}$, where $d_{t}=d t \otimes \partial / \partial t$, so the associated partial connection $\nabla^{\nu}$ on $\mathcal{A}\left(\mathcal{G}_{\widehat{F}}, \pi^{*} E\right)$ is $\nabla^{\nu}={ }^{t} \nabla^{\nu}+d_{t}$. Therefore, the curvature $\theta$ of $\nabla^{\nu}$ satisfies $i_{t}^{*}(\theta)=\theta_{t}$ the curvature of ${ }^{t} \nabla^{\nu}$, where $i_{t}: \mathcal{G} \rightarrow \mathcal{G} \times I$ is $i_{t}(x)=(x, t)$. Finally, the differential $\partial_{\nu}$ associated to $\nabla$ is $\partial_{\nu}=\partial_{t}+d_{t}$.

Denote by $\int_{I}: A_{c}(M \times I / \widehat{F}) \rightarrow A_{c}(M / F)$ integration along the fiber of $M \times I / \widehat{F}=M / F \times I \rightarrow M / F$, and let $r_{j}: M / F \rightarrow M \times I / \widehat{F}$ be given by $r_{j}(x)=(x, j)$. The usual proof shows that

$$
r_{1}^{*}-r_{0}^{*}=\int_{I} \circ d_{\widehat{H}} \pm d_{H} \circ \int_{I} \text {. }
$$

For any idempotent $(e, \lambda) \in M_{N}\left(\Psi^{-\infty}(\mathcal{G} ; E) \oplus \mathbb{C}\right)$, the associated idempotent in $M_{N}\left(\Psi^{-\infty}(\mathcal{G} \times I ; E) \oplus \mathbb{C}\right)$ is denoted by $\left(\pi^{*} e, \lambda\right)$. As

we have

$$
d_{\widehat{H}}\left((\Phi \circ \operatorname{tr})\left(\pi^{*} e\left(\delta \pi^{*} e\right)^{2 k}\right)\right)=0,
$$

$$
r_{1}^{*}\left((\Phi \circ \operatorname{tr})\left(\pi^{*} e\left(\delta \pi^{*} e\right)^{2 k}\right)\right)-r_{0}^{*}\left((\Phi \circ \operatorname{tr})\left(\pi^{*} e\left(\delta \pi^{*} e\right)^{2 k}\right)\right)= \pm d_{H}\left(\int_{I}(\Phi \circ \operatorname{tr})\left(\pi^{*} e\left(\delta \pi^{*} e\right)^{2 k}\right)\right) .
$$

Now (up to signs whose ambiguity is the same in both expressions below)

so

$$
\left(\delta \pi^{*} e\right)^{2}=\left(\begin{array}{cc}
\left(\partial_{\nu} \pi^{*} e\right)^{2}+\left(\pi^{*} e\right) \theta\left(\pi^{*} e\right) & \left(\partial_{\nu} \pi^{*} e\right) \pi^{*} e \\
\pi^{*} e\left(\partial_{\nu} \pi^{*} e\right) & \left(\pi^{*} e\right)^{2}
\end{array}\right),
$$

$$
i_{j}^{*}\left(\delta \pi^{*} e\right)^{2}=\left(\begin{array}{cc}
\left(\partial_{j} e\right)^{2}+e \theta_{j} e & \left(\partial_{j} e\right) e \\
e\left(\partial_{j} e\right) & e^{2}
\end{array}\right)=\left(\delta_{j} e\right)^{2}
$$

as $d_{j}\left(\pi^{*}(e)\right)=0$. Since $i_{j}^{*}(\theta)=\theta_{j}$, we have $i_{j}^{*}\left(\left(\delta \pi^{*} e\right)^{2 k}\right)=\left(\delta_{j} e\right)^{2 k}$. But $r_{j}^{*} \circ \Phi \circ \operatorname{tr}=\Phi \circ \operatorname{tr} \circ i_{j}^{*}$, and $i_{j}^{*}\left(\pi^{*} e\right)=e$, so

$$
r_{j}^{*}\left((\Phi \circ \operatorname{tr})\left(\pi^{*} e\left(\delta \pi^{*} e\right)^{2 k}\right)\right)=\left((\Phi \circ \operatorname{tr})\left(i_{j}^{*}\left(\pi^{*} e\left(\delta \pi^{*} e\right)^{2 k}\right)\right)\right)=(\Phi \circ \operatorname{tr})\left(e\left(\delta_{j} e\right)^{2 k}\right),
$$

and we have the theorem.

\section{Push Forward MAPS}

In this section we construct a push forward map in cohomology for oriented maps from a manifold $N$ to the space of leaves $M / F$. We will also give a geometric construction of the Connes-Skandalis push forward map [CS84] in K-theory.

Recall the following from [CS84].

Definition 5.1. A map $f: N \rightarrow M / F$ is a cocycle $\left(V_{\alpha}, f_{\alpha \beta}\right)$ on $N$ with values in $\mathcal{G}$.

Here $\mathcal{V}=\left\{V_{\alpha}\right\}$ is a locally finite open cover of $N$ and the maps $f_{\alpha \beta}: V_{\alpha} \cap V_{\beta} \rightarrow \mathcal{G}$ satisfy $f_{\alpha \beta}(x) f_{\beta \gamma}(x)=$ $f_{\alpha \gamma}(x)$ for all $x \in V_{\alpha} \cap V_{\beta} \cap V_{\gamma}$.

Note that as $f_{\alpha \alpha} f_{\alpha \alpha}=f_{\alpha \alpha}, f_{\alpha \alpha}$ maps $V_{\alpha}$ into the space of units $\mathcal{G}^{(0)}=M$. The holonomy maps along the $f_{\alpha \beta}$ provide the gluing maps to glue the local bundles $f_{\alpha \alpha}^{*} \nu \rightarrow V_{\alpha}$ together to get the pull-back vector bundle $f^{*} \nu \rightarrow N$ of the normal bundle $\nu$. The map $f$ is oriented if the vector bundle $T N \oplus f^{*} \nu$ is oriented. It is a submersion provided that each $f_{\alpha \alpha}: V_{\alpha} \rightarrow M$ is transverse to $F$. We assume that $f$ is an oriented submersion until further notice. As above, $\mathcal{U}=\left\{U_{i}\right\}$ is a finite good cover of $M$ by foliation charts for $F$. We may assume that each $\overline{f_{\alpha \alpha}\left(V_{\alpha}\right)} \subset U_{i}$ for some $i$, for if not, we may replace $\mathcal{V}$ with a locally finite refinement for which this is true. For each $\alpha$, choose $i(\alpha)$ so that $\overline{f_{\alpha \alpha}\left(V_{\alpha}\right)} \subset U_{i(\alpha)}$.

Since the $f_{\alpha \alpha}$ are submersions, they induce foliations of the $V_{\alpha}$ and because of the cocycle condition, these local foliations fit together to give a global foliation $F_{N}$ of $N$. We may assume that the $V_{\alpha}$ are foliation charts for $F_{N}$. Let $S_{\alpha}$ be a transversal of $V_{\alpha}$. The map $f_{\alpha \alpha}$ induces a map $f_{\alpha}: S_{\alpha} \rightarrow T_{i(\alpha)}$ which is a diffeomorphism onto its image, so we get a map $f_{\alpha *}: \mathcal{A}_{c}\left(S_{\alpha}\right) \rightarrow \mathcal{A}_{c}\left(T_{i(\alpha)}\right)$. Let $\omega=\left(\omega_{\alpha}\right) \in \mathcal{A}_{c}\left(N / F_{N}\right)$, be a 
Haefliger form, where $\omega_{\alpha} \in \mathcal{A}_{c}\left(S_{\alpha}\right)$. As $\omega$ has compact support and the cover $\mathcal{V}$ is locally finite, there are at most a finite number of $\alpha$ for which $\omega_{\alpha} \neq 0$. It then follows easily that $\sum_{\alpha} f_{\alpha *}$ induces a well defined map denoted $f_{*}: \mathcal{A}_{c}\left(N / F_{N}\right) \rightarrow \mathcal{A}_{c}(M / F)$. Since $f$ is oriented, $F_{N}$ is an oriented foliation on $N$. Recall the map $\int_{F_{N}}: \mathcal{A}_{c}(N) \rightarrow \mathcal{A}_{c}\left(N / F_{N}\right)$.

Definition 5.2. For an oriented submersion $f: N \rightarrow M / F, \int_{f}: \mathcal{A}_{c}(N) \rightarrow \mathcal{A}_{c}(M / F)$ is defined to be $\int_{f}=f_{*} \circ \int_{F_{N}}$.

Denote the cohomology class of any closed differential form $\alpha$ by $[\alpha]$, and similarly for Haefliger forms.

Definition 5.3. For an oriented submersion $f: N \rightarrow M / F$, the push forward map $f_{!}: \mathrm{H}_{c}^{*}(N ; \mathbb{R}) \rightarrow$ $\mathrm{H}_{c}^{*}(M / F)$ is defined by $f_{!}([\omega])=\left[\int_{f} \omega\right]$.

That this is well defined follows immediately from

\section{Proposition 5.4.}

$$
d_{H} \circ \int_{f}=\int_{f} \circ d_{N}
$$

Proof. Denote the Haefliger differentials on $M$ and $N$ by $d_{H}^{M}$ and $d_{H}^{N}$ respectively. The result then follows from the fact that $d_{H}^{M} \circ f_{*}=f_{*} \circ d_{H}^{N}$ and $d_{H}^{N} \circ \int_{F_{N}}=\int_{F_{N}} \circ d_{N}$.

This construction may be generalized as follows. Suppose that $F_{1}$ is another foliation of $N$ and that $\widehat{f}: N / F_{1} \rightarrow M / F$ is a submersion. This means that there is a submersion $f: N \rightarrow M / F$ so that the leaves of $F_{1}$ are submanifolds of the leaves $F_{N}$. We can then construct a map $\widehat{f}_{!}: \mathrm{H}_{c}^{*}\left(N / F_{1}\right) \rightarrow \mathrm{H}_{c}^{*}(M / F)$ using the techniques above. Assume that the cover $\mathcal{V}$ is a cover by foliation charts for $F_{1}$ as well as for $F_{N}$. Denote the transversal of $V_{\alpha}$ for $F_{1}$ by $\widehat{S_{\alpha}}$. We may assume that $\widehat{S}_{\alpha}$ is a fiber bundle over $S_{\alpha}$ with projection $\pi$. Integration over the fibers of these bundles defines a map $\int_{\pi}: \mathcal{A}_{c}\left(N / F_{1}\right) \rightarrow \mathcal{A}_{c}\left(N / F_{N}\right)$. We then define $\widehat{f}_{!}: \mathrm{H}_{c}^{*}\left(N / F_{1}\right) \rightarrow \mathrm{H}_{c}^{*}(M / F)$ to be $\widehat{f}_{!}([\omega])=\left[f_{*}\left(\int_{\pi} \omega\right)\right]$. Our particular case above is given by taking $F_{1}$ to be the foliation of $N$ by its points, so that $\mathrm{H}_{c}^{*}\left(N / F_{1}\right)=\mathrm{H}_{c}^{*}(N ; \mathbb{R})$.

Recall that a map $f: N \rightarrow M$ between two manifolds is oriented if the bundle $T N \oplus f^{*} T M$ is oriented. Given such a map, there is a push forward map $f_{!}: \mathrm{H}_{c}^{*}(N ; \mathbb{R}) \rightarrow \mathrm{H}_{c}^{*}(M ; \mathbb{R})$ which is defined using Poincaré duality. If $g: M \rightarrow W$ is another oriented map, then $(g \circ f) !=g ! \circ f !$. If $f$ is a submersion, $f !$ is also given by integrating over the fibers of $f$. (This is just the construction above, taking $F$ to be the foliation of $M$ by its points).

Now suppose that $f: N \rightarrow M / F$ is an arbitrary oriented map. We shall show below that there is a manifold $W$ and oriented maps $i: N \rightarrow W$ and $\widehat{f}: W \rightarrow M / F$ where $\widehat{f}$ is a submersion, and $f=\widehat{f} \circ i$.

Definition 5.5. Suppose $f: N \rightarrow M / F$ is written as $f=\widehat{f} \circ i$ as above. Define $f_{!}: \mathrm{H}_{c}^{*}(N ; \mathbb{R}) \rightarrow \mathrm{H}_{c}^{*}(M / F)$ to be the composition $f_{!}=\widehat{f}_{!} \circ i_{!}$.

Proposition 5.6. $f_{!}$is well defined. In particular, if $W_{1}, W_{2}$ are smooth manifolds, and $i_{j}: N \rightarrow W_{j}$ and $f_{j}: W_{j} \rightarrow M / F$ are oriented smooth maps where $f=f_{j} \circ i_{j}$ and each $f_{j}$ is a submersion, then $f_{1 !} \circ i_{1 !}=f_{2 !} \circ i_{2 !}$

Proof. [See [CS84], p.1169] First note that if we have maps $X \stackrel{g}{\longrightarrow} W \stackrel{f}{\longrightarrow} M / F$ where both $g$ and $f$ are oriented submersions, then $f \circ g$ is an oriented submersion, and $(f \circ g)_{!}=f_{!} \circ g_{!}$. This is just Fubini's Theorem, coupled with the fact that $g$ ! can be defined by integration over its fibers. 
In [CS84], the following commutative diagram is constructed

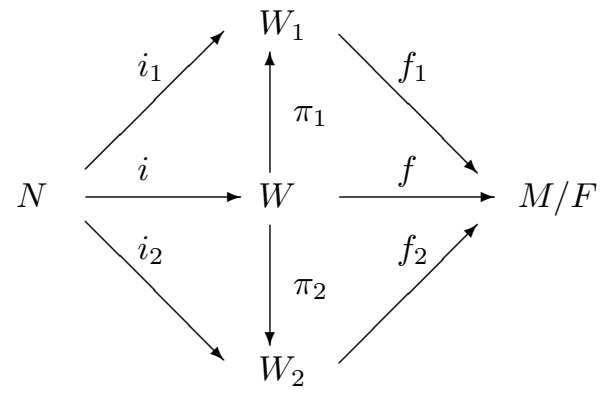

where $W$ is essentially the fibered product of $W_{1}$ and $W_{2}$ over $M / F$, and $f, \pi_{1}$ and $\pi_{2}$ are submersions. It is clear that since $i_{1}$ and $i_{2}$ are oriented, so is the fibered product map $i$. In the same way the maps $\pi_{1}$ and $\pi_{2}$ are clearly oriented. Then we have

$$
f_{1 !} \circ i_{1 !}=f_{1 !} \circ\left(\pi_{1} \circ i\right)_{!}=f_{1 !} \circ \pi_{1 !} \circ i_{!}=\left(f_{1} \circ \pi_{1}\right) ! \circ i_{!}=f_{!} \circ i_{!} .
$$

Similarly, $f_{2 !} \circ i_{2 !}=f_{!} \circ i_{!}$.

To construct a manifold $W$ and oriented maps $i: N \rightarrow W$ and $\widehat{f}: W \rightarrow M / F$ where $\widehat{f}$ is a submersion and $f=\widehat{f} \circ i$, we follow [Con81], Section 11. For the benefit of the reader, let us sketch briefly the construction of $W$. Recall the foliated microbundle $N \stackrel{i_{1}}{\rightarrow} W_{1} \stackrel{p}{\rightarrow} N$ associated to a $\Gamma_{q}$ structure $\mathcal{F}$ on $N$ introduced by Haefliger, [H58, H70]. Suppose the foliation $F$ is given by the submersions $k_{i}: U_{i} \rightarrow \mathbb{R}^{q}$ with changes of coordinates $k_{j i}: k_{i}\left(U_{i} \cap U_{j}\right) \rightarrow k_{j}\left(U_{i} \cap U_{j}\right)$. For each $i$, let $k_{i}^{-1}: \mathbb{R}^{q} \rightarrow U_{i}$ be a transversal (so $\left.k_{i} k_{i}^{-1}=I d\right)$. Note that in general, $k_{i}^{-1} k_{i}(x) \neq x$, but these points are always on the same placque of $U_{i}$. As above, assume that $\overline{f_{\alpha \alpha}\left(V_{\alpha}\right)} \subset U_{i(\alpha)}$. The $\Gamma_{q}$ structure $\mathcal{F}$ on $N$ associated to $f$ and $F$ is then given by $\mathcal{F}=\left(V_{\alpha}, h_{\alpha}, h_{\alpha \beta}^{x}\right)$, where $h_{\alpha}=k_{i(\alpha)} f_{\alpha \alpha}: V_{\alpha} \rightarrow \mathbb{R}^{q}$. For each $x \in V_{\alpha} \cap V_{\beta}$, the local diffeomorphism $h_{\alpha \beta}^{x}$ from a neighborhood of $h_{\beta}(x)$ to a neighborhood of $h_{\alpha}(x)$ is $h_{\alpha \beta}^{x}=k_{i(\alpha)} H_{\alpha \beta}^{x} k_{i(\beta)}^{-1}$, where $H_{\alpha \beta}^{x}$ is the holonomy determined by the path $f_{\alpha \beta}(x)$ from $f_{\beta \beta}(x)$ to $f_{\alpha \alpha}(x)$ acting from its domain in $k_{i(\beta)}^{-1}\left(\mathbb{R}^{q}\right)$ to its range in $k_{i(\alpha)}^{-1}\left(\mathbb{R}^{q}\right)$. Note that $\left\{h_{\alpha \beta}^{x}\right\}$ is then a cocycle. Set $\widetilde{V}_{\alpha}=V_{\alpha} \times \mathbb{R}^{q}$ and consider the disjoint union $\widetilde{V}_{\alpha}$. Each of the sets $\widetilde{V}_{\alpha}$ has the codimension $q$ foliation whose leaves are given by $V_{\alpha} \times\{r\}$ where $r \in \mathbb{R}^{q}$. Define $i_{\alpha}: V_{\alpha} \rightarrow \widetilde{V}_{\alpha}$, by $i_{\alpha}(x)=\left(x, h_{\alpha}(x)\right)$ and $p_{\alpha}: \widetilde{V}_{\alpha} \rightarrow V_{\alpha}$ by $p_{\alpha}(x, r)=x$. The space $\widetilde{W}$ is then obtained from $\coprod \widetilde{V}_{\alpha}$ by identifying $(x, s) \in \widetilde{V}_{\beta}$ with $\left(x, h_{\alpha \beta}^{x}(s)\right) \in \widetilde{V}_{\alpha}$. The maps $i_{\alpha}$ and $p_{\alpha}$ determine well defined maps, $i_{1}: N \rightarrow \widetilde{W}$, and $p: \widetilde{W} \rightarrow N$. The map $p$ is a submersion, and $p \circ i_{1}=I d$. The local foliations also fit together to give a global foliation $F_{\widetilde{W}}$ of $\widetilde{W}$, and it is easy to see that $\mathcal{F}$ is induced by $i_{1}$ and $F_{\widetilde{W}}$. Note that $\widetilde{W}$ is not a manifold in general as it will not be locally Euclidean due to the fact that the $h_{\alpha \beta}^{x}$ are only locally defined. This problem is eliminated by taking $W_{1}$ to be a sufficiently small neighborhood of the image of the $i_{1}$ in $\widetilde{W}$.

Next we construct a submersion $f_{1}: W_{1} \rightarrow M / F$ so that $f=f_{1} \circ i_{1}$. Each foliation chart $U_{i}$ has coordinates $U_{i} \simeq \mathbb{R}^{p} \times \mathbb{R}^{q}$ with the placques given by the $\mathbb{R}^{p} \times\{r\}$. Set $\widehat{V}_{\alpha}=p^{-1} V_{\alpha}$ which we may consider as a subset of $\widetilde{V}_{\alpha}=V_{\alpha} \times \mathbb{R}^{q}$. Define $f_{1_{\alpha, \alpha}}: \widehat{V}_{\alpha} \rightarrow U_{i(\alpha)}$ by

$$
f_{1 \alpha, \alpha}(x, r)=f_{\alpha, \alpha}(x)+(0, r) .
$$

We may assume that $W_{1}$ is a sufficiently small enough neighborhood of the image of $i_{1}$ in $\widetilde{W}$ so that the $f_{1 \alpha, \alpha}$ are well defined. It is obvious that each $f_{1_{\alpha, \alpha}}$ is transverse to $F$. Now suppose that $\widetilde{V}_{\alpha} \cap \widetilde{V}_{\beta} \neq \emptyset$. A point $z$ in this intersection has two different coordinates, namely $(x, r) \in V_{\alpha} \times \mathbb{R}^{q}$ and $\left(x, h_{\beta, \alpha}^{x}(r)\right) \in V_{\beta} \times \mathbb{R}^{q}$. Define $f_{1_{\beta, \alpha}}: \widehat{V}_{\alpha} \cap \widehat{V}_{\beta} \rightarrow \mathcal{G}$ by $f_{1_{\beta, \alpha}}(z)$ is the class of the leafwise path starting at $f_{1_{\alpha, \alpha}}(x, r)$, ending at the point $f_{1 \beta, \beta}\left(x, h_{\beta, \alpha}^{x}(r)\right)$ and parallel to the leafwise path determined by $f_{\beta, \alpha}(x)$. We leave it to the reader to check that this is well defined and gives a submersion $f_{1}: W_{1} \rightarrow M / F$. It is obvious that $f=f_{1} \circ i_{1}$. 
To finish the construction of $W, i$ and $\widehat{f}$, we take care of the problem of making both $\widehat{f}$ and $i$ oriented. Let $\xi \rightarrow N$ be the normal bundle of an imbedding of $N$ in some Euclidean space. Let $W=p^{*} \xi$ and denote by $\pi: W \rightarrow W_{1}$ the projection, and by $s: W_{1} \rightarrow W$ the zero section. Set

$$
i=s \circ i_{1}: N \rightarrow W \quad \text { and } \quad \widehat{f}=f_{1} \circ \pi: W \rightarrow M / F .
$$

Then $f=\widehat{f} \circ i$ since $\pi \circ s=I d$, and $\widehat{f}$ is a submersion.

It is easy to see that $i_{1}^{*} T W_{1} \simeq T N \oplus f^{*} \nu$ and $s^{*} T W=s^{*} T p^{*} \xi=T W_{1} \oplus p^{*} \xi$, so $i^{*} T W \simeq T N \oplus f^{*} \nu \oplus \xi$. Thus $T N \oplus i^{*} T W \simeq T N \oplus f^{*} \nu \oplus T N \oplus \xi$ is oriented since $f$ is oriented and $T N \oplus \xi$ is a trivial bundle.

Finally, using the fact that $i_{1}(N)$ is a deformation retract of $W_{1}$, which implies that $\left(i_{1} \circ p\right)^{*} E \simeq E$ for any bundle $E$ over $W_{1}$, we have

$$
\begin{aligned}
T W \oplus \widehat{f}^{*} \nu & \simeq \pi^{*}\left(T W_{1} \oplus p^{*} \xi\right) \oplus \pi^{*} f_{1}^{*} \nu \\
& \simeq \pi^{*}\left(p^{*}\left(T N \oplus f^{*} \nu\right) \oplus p^{*} \xi \oplus\left(i_{1} \circ p\right)^{*} f_{1}^{*} \nu\right) \\
& \simeq \pi^{*}\left(p^{*}\left(T N \oplus f^{*} \nu\right) \oplus p^{*} \xi \oplus p^{*} i_{1}^{*} f_{1}^{*} \nu\right) \\
& \simeq \pi^{*} p^{*}\left(T N \oplus f^{*} \nu \oplus \xi \oplus f^{*} \nu\right) \\
& \simeq \pi^{*} p^{*}\left(T N \oplus \xi \oplus f^{*} \nu \oplus f^{*} \nu\right),
\end{aligned}
$$

which is oriented.

We now give a geometric construction of the push forward map $f_{!}: \mathrm{K}_{c}^{0}(N) \rightarrow \mathrm{K}_{0}\left(C^{*}(M, F)\right)$ associated to the K-oriented map $f: N \rightarrow M / F$ constructed by Connes and Skandalis in [CS84]. $C^{*}(M, F)$ is Connes'

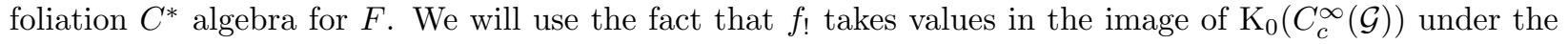
inclusion map in $K$-theory. The map $f$ is $\mathrm{K}$-oriented provided that the bundle $T^{*} N \oplus f^{*}(\nu)$ is endowed with a Spin ${ }^{c}$ structure. If $f$ is K-oriented, it is oriented. The fact that $f$ is K-oriented implies that $i$ is K-oriented, so we have the push forward map $i_{!}: \mathrm{K}_{c}^{0}(N) \rightarrow \mathrm{K}_{c}^{0}(W)$.

Denote by $\widehat{F}$ the foliation of $M \times \mathbb{R}^{2 k}$ whose leaves are of the form $L \times\{x\}$ where $L$ is a leaf of $F$ and $x \in \mathbb{R}^{2 k}$. If we denote the holonomy groupoid of $\widehat{F}$ by $\mathcal{G}^{\mathbb{R}^{2 k}}$, then $\mathcal{G}^{\mathbb{R}^{2 k}}=\mathcal{G} \times \mathbb{R}^{2 k}$, and the space of leaves of $\widehat{F}$ is $M \times \mathbb{R}^{2 k} / \widehat{F} \simeq M / F \times \mathbb{R}^{2 k}$. Let the map $\widehat{f}$ be represented by the cocycle $\left\{\hat{f}_{\alpha \beta}, V_{\alpha}\right\}$ where $\mathcal{V}=\left\{V_{\alpha}\right\}$ is a locally finite open cover of $W$. Choose an imbedding $g: W \rightarrow \mathbb{R}^{2 k}$ and let $h: W \rightarrow M \times \mathbb{R}^{2 k} / \widehat{F}$ be given by the cocycle $\left\{h_{\alpha \beta}, V_{\alpha}\right\}$ where

$$
h_{\alpha \beta}(x)=\left(\widehat{f}_{\alpha \beta}(x), g(x)\right) .
$$

Now denote by $\mathcal{U}=\left\{U_{i}\right\}$ a locally finite good cover of $M \times \mathbb{R}^{2 k}$ by foliation charts for $\widehat{F}$. As above, we may assume that each $\overline{h_{\alpha \alpha}\left(V_{\alpha}\right)} \subset U_{i}$ for some $i$, and for each $\alpha$ we may choose $i(\alpha)$ so that $\overline{h_{\alpha \alpha}\left(V_{\alpha}\right)} \subset U_{i(\alpha)}$. Note that for each $\alpha, h_{\alpha \alpha}$ is an imbedding of $V_{\alpha}$ in a transversal of the foliation $\widehat{F}$. This implies that each $h_{\alpha \beta}$ is an imbedding in a transversal of the combined foliation $\widehat{F}_{s} \oplus \widehat{F}_{r}$ of $\mathcal{G}^{\mathbb{R}^{2 k}}$.

Recall the open cover of $\mathcal{G}^{\mathbb{R}^{2 k}}$ associated to the cover $\mathcal{U}$ of $M \times \mathbb{R}^{2 k}$. For each $U_{i} \in \mathcal{U}$ choose a smooth connected transversal $T_{i} \subset U_{i}$. We may assume that these transversals are pairwise disjoint. Let $U_{i}$ and $U_{j}$ be in $\mathcal{U}$, and let $\gamma_{i j}$ be a smooth map $\gamma_{i j}: T_{i} \rightarrow \mathcal{G}^{\mathbb{R}^{2 k}}$ so that for each $y \in \operatorname{domain}\left(\gamma_{i j}\right) \subset T_{i}, s\left(\gamma_{i j}(y)\right) \in T_{i}$ and $r\left(\gamma_{i j}(y)\right) \in T_{j}$. The open subset of $\mathcal{G}^{\mathbb{R}^{2 k}}$

$$
\left(U_{i}, \gamma_{i j}, U_{j}\right)
$$

consists of the classes of all leafwise paths $\sigma$ which start in $U_{i}$, end in $U_{j}$, and if the path $\sigma$ is in the leaf of $\gamma_{i j}(y)$ then $\sigma$ is parallel to $\gamma_{i j}(y)$. We may also describe $\left(U_{i}, \gamma_{i j}, U_{j}\right)$ as the classes of all leafwise paths of the form $\gamma_{j} \circ \gamma_{i j}(y) \circ \gamma_{i}$, where $\gamma_{i}$, respectively $\gamma_{j}$, is entirely contained in the placque of $U_{i}$, respectively $U_{j}$, containing $s\left(\gamma_{i j}(y)\right)$, respectively $r\left(\gamma_{i j}(y)\right)$. Note that the image $\gamma_{i j}\left(T_{i}\right)$, denoted $T_{i j}$, is a submanifold of $\left(U_{i}, \gamma_{i j}, U_{j}\right)$ which is a transversal of the combined foliation $\widehat{F}_{s} \oplus \widehat{F}_{r}$.

The image of any map $h_{\alpha \beta}: V_{\alpha} \cap V_{\beta} \rightarrow \mathcal{G}^{\mathbb{R}^{2 k}}$ is contained in a chart of the form $\left(U_{i(\alpha)}, \gamma_{\alpha \beta}, U_{i(\beta)}\right)$ as above. We now alter the $h_{\alpha \beta}$ so that the new $h_{\alpha \beta}$ satisfy $h_{\alpha \beta}\left(V_{\alpha} \cap V_{\beta}\right) \subset \gamma_{\alpha \beta}\left(T_{i(\alpha)}\right)$. For each $x \in V_{\alpha} \cap V_{\beta}$, $h_{\alpha \beta}(x)$ may be written as the class of some $\gamma_{\beta}(x) \circ \gamma_{\alpha \beta}(y) \circ \gamma_{\alpha}(x)$, where $y \in T_{i(\alpha)}$ is in the leaf containing 
$h_{\alpha \beta}(x)$, and the paths $\gamma_{\alpha}(x), \gamma_{\beta}(x)$ are entirely contained in the placques of $U_{i(\alpha)}$ and $U_{i(\beta)}$ respectively determined by the end points of $\gamma_{\alpha \beta}(y)$. We define the new $h(x)$ to be $\gamma_{\alpha \beta}(y)$. We leave it to the reader to check that this does define a map $h: W \rightarrow M \times \mathbb{R}^{2 k} / \widehat{F}$ which is equivalent to the original $h$, and with each new $h_{\alpha \alpha}$ an immersion of $V_{\alpha}$ in a transversal of the foliation $\widehat{F}$, so each new $h_{\alpha \beta}$ is still an immersion in a transversal of the combined foliation $\widehat{F}_{s} \oplus \widehat{F}_{r}$.

Remark 5.7. Given any finite subset $\widehat{\mathcal{V}} \subset \mathcal{V}$, we may alter the map $h$ by sliding images along leaves as above and refining the cover $\mathcal{U}$ to obtain an equivalent map $h$ which satisfies, $U_{i(\alpha)} \cap U_{i(\beta)}=\emptyset$ for $V_{\alpha}, V_{\beta} \in \widehat{\mathcal{V}}$, $\alpha \neq \beta$. This will be useful later.

We now extend the map $h$ so that it is étale. Let $\nu^{g}$ be the normal bundle to the image of $T F_{W}$ in $\mathbb{R}^{2 k}$. In particular, $\nu_{x}^{g}=\left[g_{*}\left(T F_{W, x}\right)\right]^{\perp}$. Denote by $\pi_{W}: \nu^{g} \rightarrow W$ the projection and by $j: W \rightarrow \nu^{g}$ the zero section. On $\nu^{g}$, we have the open cover by the sets $\widehat{V}_{\alpha}=\pi_{W}^{-1}\left(V_{\alpha}\right)$. The map $\widehat{h}: \nu^{g} \rightarrow M \times \mathbb{R}^{2 k} / \widehat{F}$ is given by the cocycle $\left\{\widehat{h}_{\alpha \beta}, \widehat{V}_{\alpha}\right\}$, where

$$
\widehat{h}_{\alpha \beta}(x, \xi)=\left(\widehat{f}_{\alpha \beta}(x), g(x)+\xi\right) .
$$

We may choose an open neighborhood $X$ of the zero section $j(W) \subset \nu^{g}$ so that each $\widehat{h}_{\alpha \beta}$ satisfies

$$
\widehat{h}_{\alpha \beta}\left(\widehat{V}_{\alpha} \cap \widehat{V}_{\beta} \cap X\right) \subset\left(U_{i(\alpha)}, \gamma_{\alpha \beta}, U_{i(\beta)}\right),
$$

and $\widehat{h}_{\alpha \beta}$ is a diffeomorphism of $\widehat{V}_{\alpha} \cap \widehat{V}_{\beta} \cap X$ onto an open subset of the transversal $T_{i(\alpha) i(\beta)}$ of $\left(U_{i(\alpha)}, \gamma_{\alpha \beta}, U_{i(\beta)}\right)$. Set $\left.h^{X}=\left\{\widehat{h}_{\alpha \beta}, \widehat{V}_{\alpha} \cap X\right)\right\}$. Then $h^{X}: X \rightarrow M \times \mathbb{R}^{2 k} / \widehat{F}$ is an étale map, and $h=h^{X} \circ j$.

The map $j: W \rightarrow X$ is K-oriented. To see this, denote by $F_{W}$ the foliation of $W$ induced by $\widehat{f}$ and $F$, and denote its normal bundle by $\nu_{F_{W}}$. Then $T W \oplus j^{*} T X \simeq T W \oplus T W \oplus \nu^{g} \simeq(p \circ \pi)^{*}(T N \oplus \xi) \oplus \nu_{F_{W}} \oplus$ $\nu_{F_{W}} \oplus T F_{W} \oplus \nu^{g}$. As $T N \oplus \xi$ and $T F_{W} \oplus \nu^{g}$ are trivial and $\nu_{F_{W}} \oplus \nu_{F_{W}}$ has a complex structure, so is $\operatorname{Spin}^{c}$, $j$ is K-oriented. Thus we have the push forward map $j_{!}: \mathrm{K}_{c}^{0}(W) \rightarrow \mathrm{K}_{c}^{0}(X)$.

We need two more constructions in order to complete the definition of $\widehat{f}_{!}$. The first is the non-commutative space $\mathcal{R}$ associated to the open cover $\mathcal{V}_{X}=\left\{\widehat{V}_{\alpha} \cap X\right\}$ of $X$. See [Con94] Section II.2. $\alpha$. Consider the space $\mathcal{X}$ which is the disjoint union of all the $\widehat{V}_{\alpha} \cap X$. There is an obvious map $q: \mathcal{X} \rightarrow X$, and we write $z \sim z^{\prime}$ for $z, z^{\prime} \in \mathcal{X}$ if $q(z)=q\left(z^{\prime}\right)$. This is an equivalence relation and we define $\mathcal{R} \subset \mathcal{X} \times \mathcal{X}$ to be the graph of this relation with the induced topology. Note that $\mathcal{R}$ is the disjoint union of the open sets $\mathcal{R}_{\alpha \beta}=\left\{\left(z, z^{\prime}\right) \in\left(\widehat{V}_{\alpha} \cap X\right) \times\left(\widehat{V}_{\beta} \cap X\right) \mid z \sim z^{\prime}\right\} \simeq \widehat{V}_{\alpha} \cap \widehat{V}_{\beta} \cap X$. The space of compactly supported smooth functions $C_{c}^{\infty}(\mathcal{R})$ on $\mathcal{R}$ is a $*$ algebra with the operations being given by

$$
\begin{gathered}
(f * g)\left(z, z^{\prime \prime}\right)=\sum_{z \sim z^{\prime} \sim z^{\prime \prime}} f\left(z, z^{\prime}\right) g\left(z^{\prime}, z^{\prime \prime}\right) \\
f^{*}\left(z, z^{\prime}\right)=\overline{f\left(z^{\prime}, z\right) .}
\end{gathered}
$$

The $C^{*}$ algebra associated to this $*$ algebra is Morita equivalent to the $C^{*}$ algebra associated to $C_{c}^{\infty}(X)$, see Proposition 1 of [Con94]. This equivalence is effected by the $*$-algebra homomorphism $\Upsilon: C_{c}^{\infty}(X) \rightarrow$ $C_{c}^{\infty}(\mathcal{R})$, given by

$$
\Upsilon(f)=(f \circ q) \cdot a,
$$

where $a \in C_{c}^{\infty}(\mathcal{R})$ is constructed as follows. Choose a partition of unity $\left\{\phi_{\alpha}\right\}$ on $X$ subordinate to the cover $\mathcal{V}_{X}$. The value of $a$ at the point $\left(z, z^{\prime}\right) \in \mathcal{R}_{\alpha \beta}$ is

$$
a\left(z, z^{\prime}\right)=\sqrt{\phi_{\alpha}(q(z)) \phi_{\beta}\left(q\left(z^{\prime}\right)\right)} .
$$

Note that $a$ is an idempotent, i.e. $a=a^{2}=a^{*}$, and it has rank one when thought of as a matrix of functions $\left[a_{\alpha \beta}\right]$ where $a_{\alpha \beta} \in C_{c}^{\infty}\left(\mathcal{R}_{\alpha \beta}\right)$. $\Upsilon$ induces the isomorphism

$$
\Upsilon_{*}: \mathrm{K}_{c}^{*}(X)=\mathrm{K}_{0}\left(C_{c}^{\infty}(X)\right) \rightarrow \mathrm{K}_{0}\left(C_{c}^{\infty}(\mathcal{R})\right) .
$$

The second construction is the sub-groupoid $\mathcal{G}_{T} \subset \mathcal{G}^{\mathbb{R}^{2 k}}$, which consists of the classes all leafwise paths which start in the transversal $T_{i} \subset U_{i}$ and end in the transversal $T_{j} \subset U_{j}$, where $U_{i}, U_{j}$ run over all elements 
of $\mathcal{U} . \mathcal{G}_{T}$ is the union of all the transversals $T_{i j}$ of the sets $\left(U_{i}, \gamma_{i j}, U_{j}\right)$. The algebra structure on $C_{c}^{\infty}\left(\mathcal{G}_{T}\right)$ is given by

$$
\begin{gathered}
(f * g)(\gamma)=\sum_{\gamma_{1} \gamma_{0}=\gamma} f\left(\gamma_{1}\right) g\left(\gamma_{0}\right) \\
f^{*}(\gamma)=\overline{f\left(\gamma^{-1}\right)} .
\end{gathered}
$$

As above the $C^{*}$-algebras associated to $C_{c}^{\infty}\left(\mathcal{G}_{T}\right)$ and $C_{c}^{\infty}\left(\mathcal{G}^{\mathbb{R}^{2 k}}\right)$ are Morita equivalent, where the equivalence is effected as follows. For each transversal $T_{i}$, let $\varrho: D_{i} \rightarrow T_{i}$ be an imbedded normal disc bundle. We may assume that the $D_{i}$ are pairwise disjoint. Let $\ell=\operatorname{dim} \widehat{F}$, and choose a smooth function $\psi: \mathbb{D}^{\ell} \rightarrow \mathbb{R}$ with compact support so that

$$
\int_{\mathbb{D}^{\ell}} \psi^{2}=1
$$

On each $D_{i} \simeq T_{i} \times \mathbb{D}^{\ell}$ with coordinates $(x, y)$, define $\psi_{i}(x, y)=\psi(y)$. Then $\psi_{i}$ has fiber compact support and for all $x \in T_{i}$,

$$
\int_{\varrho^{-1}(x)} \psi_{i}^{2}=1
$$

Define $\Psi: C_{c}^{\infty}\left(\mathcal{G}_{T}\right) \rightarrow C_{c}^{\infty}\left(\mathcal{G}^{\mathbb{R}^{2 k}}\right)$ by

$$
\Psi(f)(\gamma)=\psi_{i}(s(\gamma)) f\left(\gamma_{i j}(y)\right) \psi_{j}(r(\gamma)),
$$

where $\gamma \in\left(U_{i}, \gamma_{i j}, U_{j}\right), s(\gamma) \in D_{i}, r(\gamma) \in D_{j}$, and $s(\gamma)$ is in the plaque determined by $y \in T_{i}$. For any $\gamma$ which does not start in some $D_{i}$ or does not end in some $D_{j}, \Psi(f)(\gamma)=0$. It is easy to check that $\Psi$ is a well defined $*$-algebra homomorphism, so it induces the map

$$
\Psi_{*}: \mathrm{K}_{0}\left(C_{c}^{\infty}\left(\mathcal{G}_{T}\right)\right) \rightarrow \mathrm{K}_{0}\left(C_{c}^{\infty}\left(\mathcal{G}^{\mathbb{R}^{2 k}}\right)\right) .
$$

Now it is quite clear that $h^{X}: X \rightarrow M \times \mathbb{R}^{2 k} / \widehat{F}$ induces a well defined map $\widehat{h}^{X}: \mathcal{R} \rightarrow \mathcal{G}_{T}$ and also a well defined *-algebra map $\widehat{h}_{*}^{X}: C_{c}^{\infty}(\mathcal{R}) \rightarrow C_{c}^{\infty}\left(\mathcal{G}_{T}\right)$, and so the map $\widehat{h}_{!}^{X}: \mathrm{K}_{0}\left(C_{c}^{\infty}(\mathcal{R})\right) \rightarrow \mathrm{K}_{0}\left(C_{c}^{\infty}\left(\mathcal{G}_{T}\right)\right)$. Recall that $C^{*}(M, F)$ and $C^{*}\left(M \times \mathbb{R}^{2 k}, \widehat{F}\right)$ are Connes' foliation $C^{*}$ algebras for $F$ and $\widehat{F}$ respectively. As $\mathcal{G}^{\mathbb{R}^{2 k}}=\mathcal{G} \times \mathbb{R}^{2 k}$, we have $\mathrm{K}_{0}\left(C^{*}\left(M \times \mathbb{R}^{2 k}, \widehat{F}\right)\right) \simeq \mathrm{K}_{0}\left(C^{*}(M, F) \widehat{\otimes} C_{0}\left(\mathbb{R}^{2 k}\right)\right)$ which by Bott periodicity is isomorphic to $\mathrm{K}_{0}\left(C^{*}(M, F)\right)$. Denote the composition of these two isomorphisms by $B: \mathrm{K}_{0}\left(C^{*}(M \times\right.$ $\left.\left.\mathbb{R}^{2 k}, \widehat{F}\right)\right) \rightarrow \mathrm{K}_{0}\left(C^{*}(M, F)\right)$.

Definition 5.8. The push forward map $f_{!}: \mathrm{K}_{c}^{0}(N) \rightarrow \mathrm{K}_{0}\left(C^{*}(M, F)\right)$ is the composition of the following maps

$$
\mathrm{K}_{c}^{0}(N) \stackrel{f_{!}^{\mathbb{R}^{2 k}}}{\longrightarrow} \mathrm{K}_{0}\left(C_{c}^{\infty}\left(\mathcal{G}^{\mathbb{R}^{2 k}}\right)\right) \longrightarrow \mathrm{K}_{0}\left(C^{*}\left(M \times \mathbb{R}^{2 k}, \widehat{F}\right)\right) \stackrel{B}{\longrightarrow} \mathrm{K}_{0}\left(C^{*}(M, F)\right),
$$

where $\mathrm{K}_{0}\left(C_{c}^{\infty}\left(\mathcal{G}^{\mathbb{R}^{2 k}}\right)\right) \rightarrow \mathrm{K}_{0}\left(C^{*}\left(M \times \mathbb{R}^{2 k}, \widehat{F}\right)\right)$ is induced by the inclusion $C_{c}^{\infty}\left(\mathcal{G}^{\mathbb{R}^{2 k}}\right) \hookrightarrow C^{*}\left(M \times \mathbb{R}^{2 k}, \widehat{F}\right)$, and $f_{!}^{\mathbb{R}^{2 k}}$ is the composite map

$$
\mathrm{K}_{c}^{0}(N) \stackrel{i_{1}}{\longrightarrow} \mathrm{K}_{c}^{0}(W) \stackrel{j_{1}}{\longrightarrow} \mathrm{K}_{c}^{0}(X) \stackrel{\Upsilon_{*}}{\longrightarrow} \mathrm{K}_{0}\left(C_{c}^{\infty}(\mathcal{R})\right) \stackrel{\widehat{h}_{!}^{X}}{\longrightarrow} \mathrm{K}_{0}\left(C_{c}^{\infty}\left(\mathcal{G}_{T}\right)\right) \stackrel{\Psi_{*}}{\longrightarrow} \mathrm{K}_{0}\left(C_{c}^{\infty}\left(\mathcal{G}^{\mathbb{R}^{2 k}}\right)\right) .
$$

The following result is proved in [CS84].

Theorem 5.9. The map $f_{!}: \mathrm{K}_{c}^{*}(N) \rightarrow \mathrm{K}_{*}\left(C^{*}(M, F)\right)$ is well defined.

Proof. This is a consequence of the homotopy invariance of $K$-theory for $C^{*}$-algebras by using the homotopy described in [ASI] [page 498]. For more details, see [CS84].

Definition 5.10. Let $f: N \rightarrow M / F$ be a $K$-oriented map and $g: W \hookrightarrow \mathbb{R}^{2 k}$ an imbedding of $W$ as above. Set

$$
\operatorname{ch}_{a}^{\mathbb{R}^{2 k}}=\int_{\mathbb{R}^{2 k}} \circ \operatorname{ch}_{a}: \mathrm{K}_{0}\left(C_{c}^{\infty}\left(\mathcal{G}^{\mathbb{R}^{2 k}}\right)\right) \longrightarrow \mathrm{H}_{c}^{*}(M / F)
$$

where $\operatorname{ch}_{a}: \mathrm{K}_{0}\left(C_{c}^{\infty}\left(\mathcal{G}^{\mathbb{R}^{2 k}}\right)\right) \rightarrow \mathrm{H}_{c}^{*}\left(M \times \mathbb{R}^{2 k} / \widehat{F}\right)$ is the Chern character associated to the foliation $\widehat{F}$ on $M \times \mathbb{R}^{2 k}$, and $\int_{\mathbb{R}^{2 k}}: \mathrm{H}_{c}^{*}\left(M \times \mathbb{R}^{2 k} / \widehat{F}\right) \simeq \mathrm{H}_{c}^{*}(M / F) \otimes \mathrm{H}_{c}^{*}\left(\mathbb{R}^{2 k} ; \mathbb{R}\right) \rightarrow \mathrm{H}_{c}^{*}(M / F)$ is integration over $\mathbb{R}^{2 k}$. 
The fact that $\operatorname{ch}_{a}^{\mathbb{R}^{2 k}} \circ f_{!}^{\mathbb{R}^{2 k}}: \mathrm{K}_{c}^{0}(N) \rightarrow \mathrm{H}_{c}^{*}(M / F)$ is well defined is an immediate consequence of our main theorem.

Theorem 5.11. Let $F$ be an oriented foliation of a compact manifold $M$, and $f: N \rightarrow M / F$ a K-oriented map. Then the following diagram commutes.

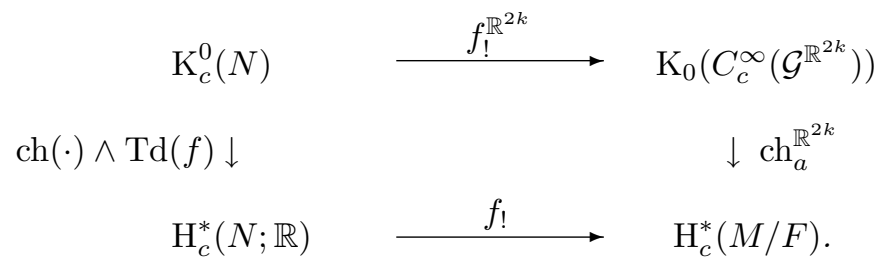

Proof. We need only show that the following diagram commutes.

$$
\begin{gathered}
\mathrm{K}_{c}^{0}(N) \stackrel{i_{!}}{\longrightarrow} \mathrm{K}_{c}^{0}(W) \stackrel{j !}{\longrightarrow} \mathrm{K}_{c}^{0}(X) \stackrel{h_{!}^{X}}{\longrightarrow} \mathrm{K}_{0}\left(C_{c}^{\infty}\left(\mathcal{G}^{\mathbb{R}^{2 k}}\right)\right) \\
\downarrow \\
\downarrow \operatorname{ch}(\cdot) \wedge \operatorname{Td}(f) \quad \downarrow \operatorname{ch}(\cdot) \wedge \operatorname{Td}(j) \quad \downarrow c h \\
\mathrm{H}_{c}^{*}(N ; \mathbb{R}) \stackrel{\operatorname{ch}_{a}}{\longrightarrow} \mathrm{H}_{c}^{*}(W ; \mathbb{R}) \stackrel{j !}{\longrightarrow} \mathrm{H}_{c}^{*}(X ; \mathbb{R}) \stackrel{h_{!}^{X}}{\longrightarrow} \mathrm{H}_{c}^{*}\left(M \times \mathbb{R}^{2 k} / \widehat{F}\right),
\end{gathered}
$$

where $h_{!}^{X}: \mathrm{K}_{c}^{0}(X) \longrightarrow \mathrm{K}_{0}\left(C_{c}^{\infty}\left(\mathcal{G}^{\mathbb{R}^{2 k}}\right)\right)$ is the composition

$$
\mathrm{K}_{c}^{0}(X) \stackrel{\Upsilon_{*}}{\longrightarrow} \mathrm{K}_{0}\left(C_{c}^{\infty}(\mathcal{R})\right) \stackrel{\widehat{h}_{!}^{X}}{\longrightarrow} \mathrm{K}_{0}\left(C_{c}^{\infty}\left(\mathcal{G}_{T}\right)\right) \stackrel{\Psi_{*}}{\longrightarrow} \mathrm{K}_{0}\left(C_{c}^{\infty}\left(\mathcal{G}^{\mathbb{R}^{2 k}}\right)\right)
$$

$\operatorname{Td}(f)=\operatorname{Td}(T N) / \operatorname{Td}\left(f^{*} \nu\right)$, and $\operatorname{Td}(j)=\operatorname{Td}(T W) / \operatorname{Td}\left(j^{*} T X\right)$. To see this, observe that

$$
f_{!}=\int_{\mathbb{R}^{2 k}} \circ h_{!}^{X} \circ j_{!} \circ i_{!}: \mathrm{H}_{c}^{*}(N ; \mathbb{R}) \rightarrow \mathrm{H}_{c}^{*}(M / F) .
$$

The classical results of [ASIII] and [CS84] imply that the second rectangle commutes up to sign. The sign comes from the definition of the Chern character, see again [ASIII]. By the same token, the left rectangle would commute if we removed the term $\wedge \operatorname{Td}(j)$ and replaced $\operatorname{Td}(f)$ by $\operatorname{Td}(i)=\operatorname{Td}(T N) / \operatorname{Td}\left(i^{*} T W\right)$. Thus to see that it does commute, we need to show that $\operatorname{Td}(f)=\operatorname{Td}(i) \wedge i^{*}(\operatorname{Td}(j))$. Let $\rho: M \times \mathbb{R}^{2 k} \rightarrow M$ be the projection and denote the normal bundle of $\widehat{F}$ by $\nu_{\widehat{F}}$. Then

$$
f=\rho \circ h^{X} \circ j \circ i
$$

so $f^{*} \nu=i^{*} j^{*} h^{X *} \rho^{*} \nu$ and $f^{*}\left(\nu \oplus \mathbb{R}^{2 k}\right)=i^{*} j^{*} h^{X *} \rho^{*}\left(\nu \oplus \mathbb{R}^{2 k}\right)=i^{*} j^{*} h^{X *}\left(\nu_{\widehat{F}}\right)=i^{*} j^{*}(T X)$, since $h^{X}$ is ètale. Thus $\operatorname{Td}\left(f^{*} \nu\right)=\operatorname{Td}\left(f^{*}\left(\nu \oplus \mathbb{R}^{2 k}\right)\right)=\operatorname{Td}\left(i^{*} j^{*} T X\right)$, so

$$
\operatorname{Td}(f)=\operatorname{Td}(T N) / \operatorname{Td}\left(i^{*} j^{*} T X\right) .
$$

As $\operatorname{Td}(i)=\operatorname{Td}(T N) / \operatorname{Td}\left(i^{*} T W\right)$ and $\operatorname{Td}(j)=\operatorname{Td}(T W) / \operatorname{Td}\left(j^{*} T X\right)$,

$$
\operatorname{Td}(i) \wedge i^{*}(\operatorname{Td}(j))=\operatorname{Td}(T N) / \operatorname{Td}\left(i^{*} j^{*} T X\right),
$$

and the left rectangle commutes.

To finish the proof we show that the third rectangle commutes by a direct computation. Let $\widehat{e}=(e, \lambda)$ be an idempotent in $M_{N}\left(C_{c}^{\infty}(X) \oplus \mathbb{C}\right)$. We now apply Remark 5.7 to the open sets of $\mathcal{V}_{X}$ covering the support of $e$ to obtain a representative of the map $h$ which satisfies the conclusions of that remark. In particular, this implies that the image of $e$ on the transversal $T_{i(\alpha)}$ under the composition $h^{X} \circ \mathrm{ch}$ is the Haefliger form for $\widehat{F}$

$$
h_{\alpha *}^{X}\left(\phi_{\alpha} \operatorname{tr}\left(e \exp \left(\frac{-(d e)^{2}}{2 i \pi}\right)\right)\right),
$$


where $d$ is the deRham differential on $X$. The degree $2 j$ part of this form is

$$
\left(\frac{-1}{2 i \pi}\right)^{j} \frac{1}{j !} h_{\alpha *}^{X}\left(\phi_{\alpha} \operatorname{tr}\left(e(d e)^{2 j}\right)\right) .
$$

The map $h_{\alpha}^{X}: \widehat{V}_{\alpha} \cap X \rightarrow T_{i(\alpha)}$ is a diffeomorphism onto its image, so we may assume that we have coordinates $\left(x_{0}, s_{0}\right)$ on $D_{i(\alpha)} \subset U_{i(\alpha)} \subset M \times \mathbb{R}^{2 k}$ of the form $\left(\widehat{V}_{\alpha} \cap X\right) \times \mathbb{D}^{\ell}$, and the transversal coordinate $x_{0}$ is given by $\left(h_{\alpha}^{X}\right)^{-1}$. Then the degree $2 j$ part of $h^{X} \operatorname{ch} e$ in these coordinates at the point $\left(x_{0}, 0\right) \in T_{i(\alpha)}$ is just

$$
\left(h^{X} \operatorname{ch} e\right)_{2 j}=\left(\frac{-1}{2 i \pi}\right)^{j} \frac{1}{j !} \phi_{\alpha} \operatorname{tr}\left(e(d e)^{2 j}\right)\left(x_{0}\right),
$$

where $d$ is now the deRham differential on $T_{i(\alpha)}$.

We now compute the degree $2 j$ part of $\operatorname{ch}_{a} h^{X} e$ on $T_{i(\alpha)}$ in these coordinates and show that it is the same as $\left(h^{X} \text { ch } e\right)_{2 j}$. As above, we have coordinates $\left(\widehat{V}_{\alpha} \cap X\right) \times \mathbb{D}^{\ell} \times \mathbb{D}^{\ell}$ on the open subset of the $\operatorname{chart}\left(U_{i(\alpha)}, \gamma_{\alpha \beta}, U_{i(\beta)}\right)$ of $\mathcal{G}^{\mathbb{R}^{2 k}}$ determined by $D_{i(\alpha)}$ and $D_{i(\beta)}$. The local coordinate functions are denoted $\left(x_{0}, s_{0}, r_{0}\right)$, where $s_{0}$ is the fiber coordinate on $D_{i(\alpha)}$ and $r_{0}$ the fiber coordinate on $D_{i(\beta)}$. Note that $h^{X} e$ can be non-zero on the chart $\left(U_{i(\alpha)}, \gamma_{\alpha \beta}, U_{i(\beta)}\right)$ only if $\widehat{V}_{\alpha} \cap \widehat{V}_{\beta} \cap X \neq \emptyset$, and it has support contained in the open subset $\left(\widehat{V}_{\alpha} \cap X\right) \times \mathbb{D}^{\ell} \times \mathbb{D}^{\ell}$. The element $h^{X} e$ in these coordinates is given by

$$
h^{X} e=\psi_{i(\beta)}\left(r_{0}\right) \psi_{i(\alpha)}\left(s_{0}\right)\left(e \sqrt{\phi_{\beta} \phi_{\alpha}}\right)\left(x_{0}\right) .
$$

If we ignore the constant $\left(\frac{-1}{2 i \pi}\right)^{j} \frac{1}{j !}$, the degree $2 j$ part of Haefliger form $\operatorname{ch}_{a} h^{X} e$ on the transversal $T_{i(\alpha)}$ is

$$
\left(\operatorname{ch}_{a} h^{X} e\right)_{2 j}=\int_{\mathbb{D}^{\ell}} \operatorname{tr}_{2}\left(h^{X} e\left(\delta\left(h^{X} e\right)\right)^{2 j}\right) d s_{0},
$$

where $\operatorname{tr}_{2}$ takes into account the $2 \times 2$ matrix $\delta\left(h^{X} e\right)$ obtained from the definition of $\delta$. An easy induction argument using the fact that $h^{X} e\left(\partial_{\nu}\left(h^{X} e\right)\right)^{2 \ell-1} h^{X} e=0$ for all $\ell>0$, shows that

$$
h^{X} e\left(\delta\left(h^{X} e\right)\right)^{2 j}=\left(\begin{array}{cc}
h^{X} e\left(\left(\partial_{\nu}\left(h^{X} e\right)\right)^{2}+\left(h^{X} e\right) \theta\left(h^{X} e\right)\right)^{j} & 0 \\
0 & 0
\end{array}\right) .
$$

Thus

$$
\left(\operatorname{ch}_{a} h^{X} e\right)_{2 j}=\int_{\mathbb{D}^{e}} \operatorname{tr}\left(h^{X} e\left(\left(\partial_{\nu}\left(h^{X} e\right)\right)^{2}+\left(h^{X} e\right) \theta\left(h^{X} e\right)\right)^{j}\right) d s_{0} .
$$

We will show below that we can choose the connection we use to define $\delta$ so that $\theta=0$, so

$$
\left(\operatorname{ch}_{a} h^{X} e\right)_{2 j}=\int_{\mathbb{D}^{\ell}} \operatorname{tr}\left(h^{X} e\left(\partial_{\nu}\left(h^{X} e\right)\right)^{2 j}\right) d s_{0} .
$$

At the point $\left(x_{0}, 0\right) \in T_{i(\alpha)}$, this is given by

$$
\int_{\mathbb{D}^{\ell}} \operatorname{tr}\left(\int_{\widetilde{L}_{s\left(\gamma_{2 j}\right)}} \cdots \int_{\widetilde{L}_{s\left(\gamma_{1}\right)}}\left(h^{X} e\right)\left(\gamma_{2 j}\right) \partial_{\nu}\left(h^{X} e\right)\left(\gamma_{2 j-1}\right) \cdots \partial_{\nu}\left(h^{X} e\right)\left(\gamma_{0}\right) d \gamma_{1} \cdots d \gamma_{2 j}\right) d s_{0}
$$

where $s\left(\gamma_{0}\right)=\left(x_{0}, s_{0}\right) \in\left(\widehat{V}_{\alpha} \cap X\right) \times \mathbb{D}^{\ell} \subset U_{i(\alpha)}$, and $\gamma_{2 j} \cdots \gamma_{0}$ is equivalent to the constant path at the point $\left(x_{0}, s_{0}\right)$. The element $\gamma_{0} \in\left(U_{i(\alpha)}, \gamma_{\alpha \beta}, U_{i\left(\beta_{1}\right)}\right)$, and for $a=1, \ldots, 2 j, \gamma_{a} \in\left(U_{i\left(\beta_{a}\right)}, \gamma_{\beta_{a} \beta_{a+1}}, U_{i\left(\beta_{a+1}\right)}\right)$, where we have the convention $U_{i\left(\beta_{2 j+1}\right)}=U_{i(\alpha)} \cdot\left(U_{i\left(\beta_{a}\right)}, \gamma_{\beta_{a} \beta_{a+1}}, U_{i\left(\beta_{a+1}\right)}\right)$ has coordinates $\left(x_{a}, s_{a}, r_{a}\right)$ from $\left(\widehat{V}_{\beta_{a}} \cap X\right) \times \mathbb{D}^{\ell} \times \mathbb{D}^{\ell}$. In these coordinates $h^{X} e$ is given by

$$
\left.h^{X} e\right|_{\left(U_{i\left(\beta_{a}\right)}, \gamma_{\beta_{a} \beta_{a+1}}, U_{i\left(\beta_{a+1}\right)}\right)}=\psi_{i\left(\beta_{a+1}\right)}\left(r_{a}\right) \psi_{i\left(\beta_{a}\right)}\left(s_{a}\right)\left(e \sqrt{\phi_{\beta_{a+1}} \phi_{\beta_{a}}}\right)\left(q\left(x_{a}\right)\right) .
$$

A straightforward computation shows that the above integral is the same as

$$
\int_{\mathbb{D} \ell} \operatorname{tr}\left(\sum_{\beta_{1} \cdots \beta_{2 j}} \int_{\mathbb{D} \ell} \cdots \int_{\mathbb{D}^{\ell}} \psi_{i(\alpha)}\left(r_{2 j}\right) \psi_{i\left(\beta_{2 j}\right)}\left(s_{2 j}\right)\left(e \sqrt{\phi_{\alpha} \phi_{\beta_{2 j}}}\right)\left(q\left(x_{2 j}\right)\right) .\right.
$$




$$
\begin{gathered}
\partial_{\nu}\left\{\psi_{i\left(\beta_{2 j}\right)}\left(r_{2 j-1}\right) \psi_{i\left(\beta_{2 j-1}\right)}\left(s_{2 j-1}\right)\left(e \sqrt{\phi_{\beta_{2 j}} \phi_{\beta_{2 j-1}}}\right)\left(q\left(x_{2 j-1}\right)\right)\right\} \wedge \cdots \wedge \\
\left.\partial_{\nu}\left\{\psi_{i\left(\beta_{1}\right)}\left(r_{0}\right) \psi_{i(\alpha)}\left(s_{0}\right)\left(e \sqrt{\phi_{\beta_{1}} \phi_{\alpha}}\right)\left(q\left(x_{0}\right)\right)\right\} d s_{1} \cdots d s_{2 j}\right) d s_{0} .
\end{gathered}
$$

The sum is over all $\beta_{a}$ with $x_{0} \in q\left(\widehat{V}_{\beta_{a}} \cap X\right)$. Note that $q\left(x_{a}\right)=x_{0}$ and $r_{a}=s_{a+1}$ for $a=0, \ldots 2 j$, where we set $s_{2 j+1}=s_{0}$. Thus the integral is

$$
\begin{gathered}
\int_{\mathbb{D}^{\ell}} \operatorname{tr}\left(\sum_{\beta_{1} \cdots \beta_{2 j}} \int_{\mathbb{D}^{\ell}} \cdots \int_{\mathbb{D}^{\ell}} \psi_{i(\alpha)}\left(s_{0}\right) \psi_{i\left(\beta_{2 j}\right)}\left(s_{2 j}\right)\left(e \sqrt{\phi_{\alpha} \phi_{\beta_{2 j}}}\right)\left(x_{0}\right) \cdot\right. \\
\partial_{\nu}\left\{\psi_{i\left(\beta_{2 j}\right)}\left(s_{2 j}\right) \psi_{i\left(\beta_{2 j-1}\right)}\left(s_{2 j-1}\right)\left(e \sqrt{\phi_{\beta_{2 j}} \phi_{\beta_{2 j-1}}}\right)\left(x_{0}\right)\right\} \wedge \cdots \wedge \\
\left.\partial_{\nu}\left\{\psi_{i\left(\beta_{1}\right)}\left(s_{1}\right) \psi_{i(\alpha)}\left(s_{0}\right)\left(e \sqrt{\phi_{\beta_{1}} \phi_{\alpha}}\right)\left(x_{0}\right)\right\} d s_{1} \cdots d s_{2 j}\right) d s_{0} .
\end{gathered}
$$

Now, the computation is local and in order to get the local expression for the transverse differential $\partial_{\nu}$ on $\left(U_{i\left(\beta_{a}\right)}, \gamma_{\beta_{a} \beta_{a+1}}, U_{i\left(\beta_{a+1}\right)}\right)$ we apply the material from the proof of Lemma 3.2 where $E_{1}$ is the $N$ dimensional trivial bundle over $M \times \mathbb{R}^{2 k}$, since $h^{X} e \in M_{N}\left(C_{c}^{\infty}\left(\mathcal{G}^{\mathbb{R}^{2 k}}\right)\right)$. We choose the connection on $E_{1}$ to be the canonical flat connection. Then $\nabla^{\nu}$ on $\left(U_{i\left(\beta_{a}\right)}, \gamma_{\beta_{a} \beta_{a+1}}, U_{i\left(\beta_{a+1}\right)}\right)$ is given by

$$
\nabla^{\nu}=d_{x_{a}}+d_{s_{a}}
$$

and $\theta=\left(\nabla^{\nu}\right)^{2}=0$ as promised above. Locally

$$
\partial_{\nu}\left(h^{X} e\right)=\left(d_{x_{a}}+d_{s_{a}}\right)\left(h^{X} e\right) .
$$

Using these facts, the fact that we may identify each $d_{x_{a}}$ with $d$ on $T_{i(\alpha)}$, as well as the fact that $\int_{\mathbb{D} \ell} \psi_{i} d_{s_{i}} \psi_{i}=$ 0 , the integral becomes

$$
\begin{gathered}
\int_{\mathbb{D} \ell} \operatorname{tr}\left(\sum_{\beta_{1} \cdots \beta_{2 j}} \int_{\mathbb{D}^{\ell}} \ldots \int_{\mathbb{D}^{\ell}} \psi_{i(\alpha)}\left(s_{0}\right) \psi_{i\left(\beta_{2 j}\right)}\left(s_{2 j}\right)\left(e \sqrt{\phi_{\alpha} \phi_{\beta_{2 j}}}\right)\left(x_{0}\right) .\right. \\
\left\{\psi_{i\left(\beta_{2 j}\right)}\left(s_{2 j}\right) \psi_{i\left(\beta_{2 j-1}\right)}\left(s_{2 j-1}\right) d\left(e \sqrt{\phi_{\beta_{2 j}} \phi_{\beta_{2 j-1}}}\right)\left(x_{0}\right)\right\} \wedge \cdots \wedge \\
\left.\left\{\psi_{i\left(\beta_{1}\right)}\left(s_{1}\right) \psi_{i(\alpha)}\left(s_{0}\right) d\left(e \sqrt{\phi_{\beta_{1}} \phi_{\alpha}}\right)\left(x_{0}\right)\right\} d s_{1} \cdots d s_{2 j}\right) d s_{0} .
\end{gathered}
$$

Since $\int_{\mathbb{D}^{\ell}} \psi_{i}^{2}=1$, this equals

$$
\operatorname{tr}\left(\sum_{\beta_{1} \cdots \beta_{2 j}} e \sqrt{\phi_{\alpha} \phi_{\beta_{2 j}}} d\left(e \sqrt{\phi_{\beta_{2 j}} \phi_{\beta_{2 j-1}}}\right) \wedge \cdots \wedge d\left(e \sqrt{\phi_{\beta_{1}} \phi_{\alpha}}\right)\right)\left(x_{0}\right)
$$

which equals

$$
\operatorname{tr}\left(\sum_{\beta_{1} \cdots \beta_{2 j}} \phi_{\alpha} \phi_{\beta_{2 j}} \cdots \phi_{\beta_{1}} e d e \wedge \cdots \wedge d e\right)\left(x_{0}\right)+\text { other terms. }
$$

As $\sum_{\beta} \phi_{\beta}=1$ the first term above is just

$$
\phi_{\alpha} \operatorname{tr}\left(e(d e)^{2 j}\right)\left(x_{0}\right)
$$

so we need only show that the "other terms" are zero in Haefliger cohomology. These terms come in several types.

(1) Those which have a repeated $d \phi_{\beta_{i}}$, such as

$$
\operatorname{tr}\left(\sum_{\beta_{1} \cdots \beta_{2 j}} e \sqrt{\phi_{\alpha}} \frac{d \phi_{\beta_{2 j}}}{2 \sqrt{\phi_{\beta_{2 j}}}} \wedge e \frac{d \phi_{\beta_{2 j}}}{2 \sqrt{\phi_{\beta_{2 j}}}} \sqrt{\phi_{\beta_{2 j-1}}} \wedge \cdots \wedge d\left(e \sqrt{\phi_{\beta_{1}} \phi_{\alpha}}\right)\right),
$$

which are obviously zero as forms. 
(2) Those which have a $d \phi_{\beta_{i}}$ which is not repeated, such as

$$
\operatorname{tr}\left(\sum_{\beta_{1} \cdots \beta_{2 j}} e \sqrt{\phi_{\alpha}} \frac{1}{2} d \phi_{\beta_{2 j}} \wedge e \frac{1}{2} d \phi_{\beta_{2 j-1}} \wedge \cdots \wedge d\left(e \sqrt{\phi_{\beta_{1}} \phi_{\alpha}}\right)\right) .
$$

These are zero as forms since they equal

$$
\operatorname{tr}\left(\sum_{\beta_{1} \cdots \beta_{2 j-1}} e \sqrt{\phi_{\alpha}} \frac{1}{2} d\left(\sum_{\beta_{2 j}} \phi_{\beta_{2 j}}\right) \wedge e \frac{1}{2} d \phi_{\beta_{2 j-1}} \wedge \cdots \wedge d\left(e \sqrt{\phi_{\beta_{1}} \phi_{\alpha}}\right)\right),
$$

and $\sum_{\beta_{2 j}} \phi_{\beta_{2 j}}=1$ near $x_{0}$.

(3) Those with no $d \phi_{\beta_{i}}$, in which case we have the form

$$
\operatorname{tr}\left(\sum_{\beta_{1} \cdots \beta_{2 j}} e \sqrt{\phi_{\beta_{2 j}}} d e \sqrt{\phi_{\beta_{2 j}} \phi_{\beta_{2 j-1}}} \wedge \cdots \wedge d e \sqrt{\phi_{\beta_{2}} \phi_{\beta_{1}}} \wedge e \sqrt{\phi_{\beta_{1}}} \wedge \frac{1}{2} d \phi_{\alpha}\right) .
$$

The Haefliger class determined by all these local forms is zero since it is the local image under $h^{X}$ of the global form on $X$

$$
\operatorname{tr}\left(\sum_{\beta_{1} \cdots \beta_{2 j}} e \sqrt{\phi_{\beta_{2 j}}} d e \sqrt{\phi_{\beta_{2 j}} \phi_{\beta_{2 j-1}}} \wedge \cdots \wedge d e \sqrt{\phi_{\beta_{2}} \phi_{\beta_{1}}} \wedge e \sqrt{\phi_{\beta_{1}}} \wedge \frac{1}{2} d\left(\sum_{\alpha} \phi_{\alpha}\right)\right)
$$

which is zero since $\sum_{\alpha} \phi_{\alpha}=1$.

Remark 5.12. As pointed out to us by G. Skandalis, the results of [Con86] hold for any holonomy invariant current. Therefore, the pairing of the Chern-Connes character $\mathrm{ch}_{a}$ with any holonomy invariant current extends to the $K$-theory of the foliation $C^{*}$-algebra. Hence, the proof above shows using the ConnesSkandalis index theorem in the $K$-theory of the foliation $C^{*}$-algebra that $\mathrm{ch}_{a}^{\mathbb{R}^{2 k}} \circ f_{!}^{\mathbb{R}^{2 k}}$ is given by

$$
\operatorname{ch}_{a}^{\mathbb{R}^{2 k}} \circ f_{!}^{\mathbb{R}^{2 k}}=\operatorname{ch}_{a} \circ f_{!}^{a n},
$$

where $f_{!}^{a n}$ is the analytic shrieck map described in [CS84].

\section{ThE INDEX THEOREM}

In this section, we compute the Chern character of the topological index of any class $u \in \mathrm{K}_{c}^{0}(T F)$. This permits us to translate the Connes-Skandalis index theorem [CS84] to Haefliger cohomology and yields an alternative proof of the longitudinal index theorem in Haefliger cohomology for all longitudinal pseudodifferential operators, compare [HL99, GL03, GL05].

We first describe the Chern character of the topological index, i.e. the homomorphism $\operatorname{ch}_{a}\left(\operatorname{Ind}_{t}\right):$ $\mathrm{K}_{c}^{0}(T F) \rightarrow \mathrm{H}_{c}^{*}(M / F)$. Choose a smooth imbedding $g: M \hookrightarrow \mathbb{R}^{2 k}$. Denote by $\pi_{F}: T F \rightarrow M$ the projection, and by $\pi_{N}: N \rightarrow M$ the normal bundle to $T F$ in $\mathbb{R}^{2 k}$. Let $j: M \hookrightarrow N$ be the zero section of $N$ and $f: T F \rightarrow N$ the composite map

$$
f=j \circ \pi_{F} .
$$

The map $f$ is $K$-oriented by a complex structure since $T(T F) \simeq \pi_{F}^{*}(T F \oplus T M)$ and $f^{*}(T N) \simeq \pi_{F}^{*}(N \oplus T M)$, so

$$
T(T F) \oplus f^{*}(T N) \simeq \pi_{F}^{*}(T F \oplus N \oplus T M \oplus T M) \simeq \pi_{F}^{*}\left(g^{*}\left(T \mathbb{R}^{2 k}\right) \oplus T M \oplus T M\right) .
$$

Therefore the Atiyah-Singer shriek map $f_{!}: \mathrm{K}_{c}^{0}(T F) \rightarrow \mathrm{K}_{c}^{0}(N)$ is well defined, see for instance [ASI]. Moreover the Todd class of $f$ is given by

$$
\begin{gathered}
\operatorname{Td}(f)=\operatorname{Td}(T(T F)) / \operatorname{Td}\left(f^{*}(T N)\right)=\operatorname{Td}\left(\pi_{F}^{*}(T F \oplus T M)\right) / \operatorname{Td}\left(\pi_{F}^{*}(N \oplus T M)\right)= \\
\pi_{F}^{*}(\operatorname{Td}(T F) / \operatorname{Td}(N))=\pi_{F}^{*}(\operatorname{Td}(T F) \cdot \operatorname{Td}(T F))=\pi_{F}^{*}(\operatorname{Td}(T F \oplus T F))=\pi_{F}^{*}(\operatorname{Td}(T F \otimes \mathbb{C})),
\end{gathered}
$$

since $1 / \operatorname{Td}(N)=\operatorname{Td}(T F)$ as $N \oplus F \simeq M \times \mathbb{R}^{2 k}$. 
Recall the foliation $\widehat{F}$ of $M \times \mathbb{R}^{2 k}$ and its holonomy groupoid $\mathcal{G}^{\mathbb{R}^{2 k}}=\mathcal{G} \times \mathbb{R}^{2 k}$. The bundle $N$ may be identified with an open transversal of $\widehat{F}$ by the map $i: N \rightarrow M \times \mathbb{R}^{2 k}$ where

$$
i(x, \xi)=(x, g(x)+\xi) .
$$

On a suitable neighborhood of the zero section, this gives an open transversal and we may assume that the neighborhood is in fact all of $N$. Thus we have the map

$$
i_{!}: \mathrm{K}_{c}^{0}(N) \longrightarrow \mathrm{K}_{0}\left(C_{c}^{\infty}\left(\mathcal{G}^{\mathbb{R}^{2 k}}\right)\right) .
$$

The inclusion $\rho^{\mathbb{R}^{2 k}}: C_{c}^{\infty}\left(\mathcal{G}^{\mathbb{R}^{2 k}}\right) \hookrightarrow C^{*}\left(M \times \mathbb{R}^{2 k}, \widehat{F}\right)$ gives $\rho_{*}^{\mathbb{R}^{2 k}}: \mathrm{K}_{0}\left(C_{c}^{\infty}\left(\mathcal{G}^{\mathbb{R}^{2 k}}\right)\right) \rightarrow \mathrm{K}\left(C^{*}\left(M \times \mathbb{R}^{2 k}, \widehat{F}\right)\right)$. Finally, we have the Bott isomorphism $B: \mathrm{K}_{0}\left(C^{*}\left(M \times \mathbb{R}^{2 k}, \widehat{F}\right)\right) \simeq \mathrm{K}_{0}\left(C^{*}(M, F) \widehat{\otimes} C_{0}\left(\mathbb{R}^{2 k}\right)\right) \rightarrow \mathrm{K}_{0}\left(C^{*}(M, F)\right)$.

Definition 6.1. [CS84] The topological index morphism is the composite map

$$
\operatorname{Ind}_{t}=B \circ \rho_{*}^{\mathbb{R}^{2 k}} \circ i_{!} \circ f_{!}: \mathrm{K}_{c}^{0}(T F) \longrightarrow \mathrm{K}_{0}\left(C^{*}(M, F)\right) .
$$

$\operatorname{Ind}_{t}$ is well defined, see [CS84]. Moreover, if $\pi: T F \rightarrow M / F$ is the natural map, then $\pi$ is a $K$-oriented submersion and it is clear that $i_{!} \circ f_{!}=\pi_{!}^{\mathbb{R}^{2 k}}$. We therefore denote by $\operatorname{ch}_{a}\left(\operatorname{Ind}_{t}\right)$ the map

$$
\operatorname{ch}_{a}\left(\operatorname{Ind}_{t}\right)=\operatorname{ch}_{a}^{\mathbb{R}^{2 k}} \circ \pi_{!}^{\mathbb{R}^{2 k}}: \mathrm{K}_{c}^{0}(T F) \longrightarrow \mathrm{H}_{c}^{*}(M / F) .
$$

Theorem 6.2. For any $u \in \mathrm{K}_{c}^{0}(T F), \mathrm{ch}_{a}\left(\operatorname{Ind}_{t}\right)(u)$, the algebraic Chern character of the topological index of $u$, is given by

$$
\operatorname{ch}_{a}\left(\operatorname{Ind}_{t}\right)(u)=(-1)^{p} \int_{F} \pi_{F !}(\operatorname{ch}(u)) \operatorname{Td}(T F \otimes \mathbb{C}) \quad \in \mathrm{H}_{c}^{*}(M / F),
$$

where $\pi_{F !}: H_{c}^{*}(T F ; \mathbb{R}) \rightarrow \mathrm{H}^{*}(M ; \mathbb{R})$ is integration along the fibers, and $\mathrm{ch}: \mathrm{K}_{c}^{0}(T F) \rightarrow H_{c}^{*}(T F)$ is the usual Chern character.

Proof. As $i: N \rightarrow M \times \mathbb{R}^{2 k}$ is a transversal to $\widehat{F}$, we may regard $i$ as a map from $N$ to $M \times \mathbb{R}^{2 k} / \widehat{F}$ and we have the push forward map

$$
i_{!}: \mathrm{H}_{c}^{*}(N ; \mathbb{R}) \rightarrow \mathrm{H}_{c}^{*}\left(M \times \mathbb{R}^{2 k} / \widehat{F}\right) .
$$

The essence of the theorem is that the following diagram commutes up to sign.

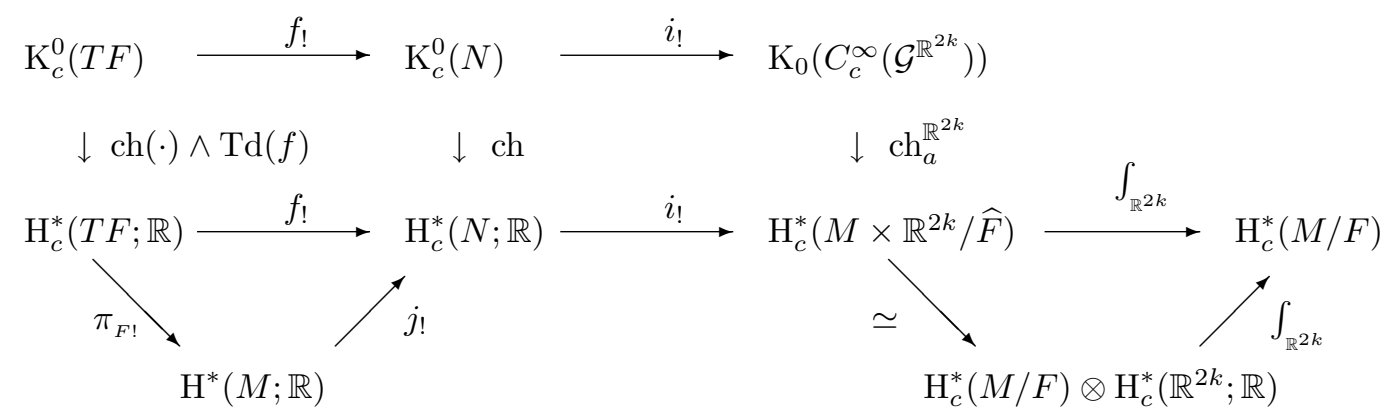

The classical results of [ASIII] and [CS84] imply that the left rectangle commutes up to sign. The sign comes from the definition of the Chern character and can be fixed by inspecting trivial examples, see again [ASIII]. The triangles commute by definition. The fact that the rest of the diagram commutes is just Theorem 5.11. Now observe that

$$
\int_{\mathbb{R}^{2 k}} \circ \simeq \circ i_{!} \circ j_{!}=\int_{F}
$$

The theorem follows immediately since $\operatorname{Td}(f)=\pi_{F}^{*} \operatorname{Td}(T F \otimes \mathbb{C})$.

We now discuss the relationship of the results here with the Connes-Skandalis index theorem, and answer a question asked in [He95]. As in Section 3, let $P$ be a uniformly supported elliptic pseudo-differential $\mathcal{G}$-operator acting on the bundle $E=r^{*}\left(E_{1}\right)$. Restricting $P$ to the holonomy cover of a leaf $L$ of $F$ gives a $\mathcal{G}(L)$-invariant pseudo-differential operator, where $\mathcal{G}(L)$ is the holonomy group of the leaf $L$. Therefore, the principal symbol of $P$ can be defined using the induced operator on $E_{1} \mid L$. We get in this way a section $\sigma(P)$ 
of the bundle $\operatorname{Hom}\left(\pi_{F}^{*} E_{1}\right)$ over $T F$. An element $P \in \Psi^{\infty}(\mathcal{G} ; E)$ is elliptic if $\sigma(P)(\xi):\left(\pi_{F}^{*} E_{1}\right)_{\xi} \rightarrow\left(\pi_{F}^{*} E_{1}\right)_{\xi}$ is an isomorphism for all $\xi \neq 0, \xi \in T F$. Denote by $\operatorname{Ind}_{a}: \mathrm{K}_{c}^{0}(T F) \longrightarrow \mathrm{K}_{0}\left(C_{c}^{\infty}(\mathcal{G})\right)$ Connes-Skandalis analytic index map [CS84]. This map associates to an element $u$ the analytic index $\operatorname{Ind}_{a}(P)$ of any elliptic pseudo-differential $\mathcal{G}$-operator $P$ with symbol $\sigma(P)=u$. The construction of $P$ from $u$ is given in [Con79]. Denote by

$$
\rho_{*}: \mathrm{K}_{0}\left(C_{c}^{\infty}(\mathcal{G})\right) \longrightarrow \mathrm{K}_{0}\left(C^{*}(M, F)\right)
$$

the homomorphism induced by the inclusion map $\rho: C_{c}^{\infty}(\mathcal{G}) \hookrightarrow C^{*}(M, F)$. Note that $\rho_{*}$ need not be injective. Recall the Connes-Skandalis leafwise index theorem.

Theorem 6.3. $[\mathrm{CS} 84] \quad \rho_{*} \circ \operatorname{Ind}_{a}=\operatorname{Ind}_{t}: \mathrm{K}_{c}^{0}(T F) \longrightarrow \mathrm{K}_{0}\left(C^{*}(M, F)\right)$.

Let $\mathcal{A}$ be any *-subalgebra of $C^{*}\left(M \times \mathbb{R}^{2 k}, \widehat{F}\right)$ which contains the smooth convolution algebra $C_{c}^{\infty}\left(\mathcal{G}^{\mathbb{R}^{2 k}}\right)$, and denote by $\rho^{\mathcal{A}}: \mathcal{A} \rightarrow C^{*}\left(M \times \mathbb{R}^{2 k}, \widehat{F}\right)$ the inclusion. An example of such algebra is the algebra of operators in $C^{*}\left(M \times \mathbb{R}^{2 k}, \widehat{F}\right)$ with smooth kernels having superexponential decay, see [HL02]. In that case, the Chern character $\operatorname{ch}_{a}^{\mathbb{R}^{2 k}}$ extends to the $K$-theory of $\mathcal{A}$.

Theorem 6.4. Suppose that the Chern character $\operatorname{ch}_{a}^{\mathbb{R}^{2 k}}$ extends to $\mathrm{K}(\mathcal{A})$ and that $\operatorname{Ker}\left(\rho_{*}^{\mathcal{A}}\right) \subset \operatorname{Ker}\left(\operatorname{ch}_{a}^{\mathbb{R}^{2 k}}\right)$. Then for all $u \in \mathrm{K}_{c}^{0}(T F)$,

$$
\operatorname{ch}_{a}\left(\operatorname{Ind}_{t}\right)(u)=\operatorname{ch}_{a}\left(\operatorname{Ind}_{a}(u)\right) .
$$

Proof. The Connes-Skandalis index theorem gives

$$
B^{-1} \circ \rho_{*} \circ \operatorname{Ind}_{a}=\rho_{*}^{\mathbb{R}^{2 k}} \circ \pi_{!}^{\mathbb{R}^{2 k}} .
$$

Denote by $\varphi: \mathrm{K}_{0}\left(C_{c}^{\infty}(\mathcal{G})\right) \rightarrow \mathrm{K}_{0}\left(C_{c}^{\infty}\left(\mathcal{G}^{\mathbb{R}^{2 k}}\right)\right)$ the Bott homomorphism. Then

$$
B^{-1} \circ \rho_{*}=\rho_{*}^{\mathbb{R}^{2 k}} \circ \varphi \text {. }
$$

Denote by $i_{*}^{\mathbb{R}^{2 k}}: \mathrm{K}_{0}\left(C_{c}^{\infty}\left(\mathcal{G}^{\mathbb{R}^{2 k}}\right) \rightarrow \mathrm{K}_{0}(\mathcal{A})\right.$ the map induced by the inclusion, and set

$$
\operatorname{Ind}_{a}^{\mathcal{A}}(u)=\left(i_{*}^{\mathbb{R}^{2 k}} \circ \varphi\right)\left(\operatorname{Ind}_{a}(u)\right) \quad \text { and } \quad \operatorname{Ind}_{t}^{\mathcal{A}}(u)=\left(i_{*}^{\mathbb{R}^{2 k}} \circ \pi_{!}^{\mathbb{R}^{2 k}}\right)(u) \text {. }
$$

These satisfy

$$
\rho_{*}^{\mathcal{A}}\left(\operatorname{Ind}_{t}^{\mathcal{A}}(u)\right)=\rho_{*}^{\mathcal{A}}\left(\operatorname{Ind}_{a}^{\mathcal{A}}(u)\right)
$$

so

$$
\operatorname{Ind}_{a}^{\mathcal{A}}(u)-\operatorname{Ind}_{t}^{\mathcal{A}}(u) \in \operatorname{Ker}\left(\rho_{*}^{\mathcal{A}}\right)
$$

and

$$
\operatorname{ch}_{a}^{\mathbb{R}^{2 k}}\left(\operatorname{Ind}_{t}^{\mathcal{A}}(u)\right)=\operatorname{ch}_{a}^{\mathbb{R}^{2 k}}\left(\operatorname{Ind}_{a}^{\mathcal{A}}(u)\right) \in \mathrm{H}_{c}^{*}\left(M \times \mathbb{R}^{2 k} / \widehat{F}\right) .
$$

Integrating over $\mathbb{R}^{2 k}$, we get by definition of the Chern character of the topological index

$$
\operatorname{ch}_{a}\left(\operatorname{Ind}_{t}\right)(u)=\int_{\mathbb{R}^{2 k}} \operatorname{ch}_{a}^{\mathbb{R}^{2 k}}\left(\operatorname{Ind}_{t}^{\mathcal{A}}(u)\right)=\int_{\mathbb{R}^{2 k}} \operatorname{ch}_{a}^{\mathbb{R}^{2 k}}\left(\operatorname{Ind}_{a}^{\mathcal{A}}(u)\right)=\operatorname{ch}_{a}\left(\operatorname{Ind}_{a}(u)\right) .
$$

From the deep extension theorem of [Con86], it follows that $\operatorname{Ker}\left(\rho_{*}^{\mathbb{R}^{2 k}}\right) \subset \operatorname{Ker}\left(\operatorname{ch}_{a}^{\mathbb{R}^{2 k}}\right)$ i.e. Theorem 6.4 holds for the case $\mathcal{A}=C_{c}^{\infty}\left(\mathcal{G}^{\mathbb{R}^{2 k}}\right)$. Therefore, we deduce from our computation of the Chern character of the topological index, the cohomological formula for all elliptic pseudodifferential operators on foliations.

Corollary 6.5. For any $u \in \mathrm{K}_{c}^{0}(T F), \operatorname{ch}_{a}\left(\operatorname{Ind}_{t}\right)(u)=\operatorname{ch}_{a}\left(\operatorname{Ind}_{a}(u)\right)$ and

$$
\operatorname{ch}_{a}\left(\operatorname{Ind}_{a}(u)\right)=(-1)^{p} \int_{F} \pi_{F !}(\operatorname{ch}(u)) \operatorname{Td}(T F \otimes \mathbb{C}) \quad \in \mathrm{H}_{c}^{*}(M / F) .
$$

Thus we have the following. 
Theorem 6.6. Suppose that $P$ is a leafwise elliptic pseudodifferential operator defined along the leaves of a foliation. Then

in Haefliger cohomology.

$$
\operatorname{ch}_{a}\left(\operatorname{Ind}_{t}\right)([\sigma(P)])=\operatorname{ch}_{a}\left(\operatorname{Ind}_{a}(P)\right)
$$

A particularly interesting case is that of $\operatorname{spin}^{c}$ Dirac operators. Compare with [GL03, GL05]:

Corollary 6.7. Suppose $T F$ admits a Spin ${ }^{c}$ structure and denote by $L$ the line bundle corresponding to that structure. Let $\alpha: \mathrm{K}^{0}(M) \rightarrow \mathrm{K}_{c}^{0}(T F)$ be the corresponding Thom isomorphism. Then for any $u \in \mathrm{K}^{0}(M)$,

$$
\operatorname{ch}_{a}\left(\operatorname{Ind}_{a}(\alpha(u))\right)=\int_{F} \hat{A}(T F) e^{c_{1}(L) / 2} \operatorname{ch}(u) \in \mathrm{H}_{c}^{*}(M / F)
$$

where $c_{1}(L)$ is the Chern class of $L, \hat{A}(T F)$ is the $\hat{A}$-genus of $T F$, [LM89], and $\operatorname{ch}: \mathrm{K}^{0}(M) \rightarrow \mathrm{H}^{*}(M ; \mathbb{R})$ is the usual Chern character.

Proof. The Thom isomorphism $\alpha$ is exactly the Gysin shriek map corresponding to the $K$-oriented inclusion of the zero section of $T F$ [ASI]. Therefore, by functoriality of the Gysin shriek maps [CS84], the composite map $f ! \circ \alpha$ coincides with the Gysin shriek $j$ ! map corresponding to the inclusion of the zero section $j: M \hookrightarrow N$ of the $K$-oriented bundle $N$. Hence

$$
\operatorname{ch}_{a}\left(\operatorname{Ind}_{t}\right)(\alpha(u))=(-1)^{p} \int_{F} \operatorname{Td}(j) \operatorname{ch}(u) .
$$

Now an inspection of the cohomology class $\operatorname{Td}(j)$ shows that

$$
\operatorname{Td}(j)=1 / \operatorname{Td}(N)=\operatorname{Td}(T F)
$$

where here Td means the Todd class of the $\operatorname{Spin}^{c}$ bundle, see [LM89, BD81]. But the Spin ${ }^{c}$ Todd class of the bundle $T F$ is precisely $(-1)^{p} \hat{A}(T F) e^{c_{1}(L) / 2}$ [BD81].

As an immediate corollary, we have an index formula for twisted spin Dirac operators [LM89].

Corollary 6.8. Assume that the foliation $F$ is spin and let $D$ be a leafwise Dirac operator associated to the spin structure on TF and complex vector bundle $E$ over $M$. Then

$$
\operatorname{ch}_{a}\left(\operatorname{Ind}_{a}([\sigma(D)])\right)=\int_{F} \widehat{A}(T F) \operatorname{ch}(E) \quad \in \mathrm{H}_{c}^{*}(M / F) .
$$

Proof. The integrand of Theorem 6.2 is known to be equal to $\widehat{A}(T F) \operatorname{ch}(E)$ for such Dirac operators by classical characteristic computations, [ASIII].

\section{REFERENCES}

[A75] M. F. Atiyah, Elliptic operators, discrete groups and von Neumann algebras, Asterisque 32/33 (1976) 43-72.

[ASI] M. F. Atiyah and I. Singer, The index of elliptic operators I, Ann. of Math. 87 1965, 484-530.

[ASIII] M. F. Atiyah and I. Singer, The index of elliptic operators III, Ann. of Math. 87 1965, 564-604.

[BC84] P. Baum and A. Connes, K-theory for Lie groups and foliations, Enseign. Math. 46 (2000), no. 1-2, 3-42.

[BCH90] P. Baum, A. Connes and N. Higson, Classifying space for proper actions and $K$-theory of group $C^{*}$-algebras. $C^{*-}$ algebras: 1943-1993 (San Antonio, TX, 1993), 240-291, Contemp. Math., 167, Amer. Math. Soc., Providence, RI, 1994.

[BD81] P. Baum and R. Douglas, K-homology and index theory, Operator algebras and applications, Part I, Proc. Sympos. Pure Math 38, Amer. Math. Soc., (1982) 117-173.

[BN03] M-T. Benameur and V. Nistor, Residues and an index theorem for foliations, to appear in Math. Scand.

[Con79] A. Connes, Sur la théorie de l'intégration non commutative. Lect. Notes in Math. 725, 1979.

[Con81] A. Connes. A survey of foliations and operator algebras. Operator algebras and applications, Part I, Proc. Sympos. Pure Math 38, Amer. Math. Soc., (1982) 521-628.

[Con86] A. Connes. Cyclic cohomology and the transverse fundamental class of a foliation, Geometric methods in operator algebras (Kyoto, 1983), Res. Notes in Mathematics 123, Pitman, London, (1986) 52-144.

[Con94] A. Connes, Noncommutative Geometry, Academic Press, New York, 1994.

[CM91] A. Connes and H. Moscovici, Cyclic cohomology and the Novikov conjecture for hyperbolic groups, Topology 29 (1990) 345-388.

[CS84] A. Connes, and G. Skandalis. The longitudinal index theorem for foliations, Publ. RIMS Kyoto 20 (1984) 1139-1183. 
[GL03] A. Gorokhovsky, and J. Lott. Local index theory over étale groupoids, J. reine angew Math 5600 (2003) $151-198$.

[GL05] A. Gorokhovsky, and J. Lott. Local index theory over foliation groupoids, preprint (2005).

[H58] A. Haefliger. Structures feuilletées et cohomologie à valeur dans un faisceau de groupoïdes, Comm. Math. Helv. 32 (1958) 248-329.

[H70] A. Haefliger. Feuilletages sur les variétés ouvertes, Topology, 9 (1970) 183-194.

[H80] A. Haefliger. Some remarks on foliations with minimal leaves, J. Diff. Geo. 15 (1980) $269-284$.

[He95] J. L. Heitsch, Bismut superconnections and the Chern character for Dirac operators on foliated manifolds. K-Theory 9 (1995), no. 6, 507-528.

[HL90] J. L. Heitsch and C. Lazarov. A Lefschetz theorem for foliated manifolds, Topology, 29 (1990) $127-162$.

[HL99] J. L. Heitsch and C. Lazarov. A general families index theorem, K-Theory, 18 (1999) $181-202$.

[HL02] J. L. Heitsch and C. Lazarov. Riemann-Roch-Grothendieck and torsion for foliations J. Geo. Anal. 12 (2002) 437-468.

[HK99] N. Higson and G. Kasparov, E-theory and KK-theory for groups which act properly and isometrically on Hilbert space, Invent. Math. 144 (2001), no. 1, 23-74.

[K88] G. Kasparov, Equivariant KK-theory and the Novikov conjecture, Inv. Math. 91 (1988) $147-201$.

[LM89] H. B. Lawson and M.-L. Michelson, Spin Geometry, Princeton Math. Series 38, Princeton, 1989.

[NWX96] V. Nistor, A. Weinstein and P. Xu, Pseudodifferential operators on differential groupoids Pacific J. Math. 189 (1999), no. 1, 117-152.

[T97] P. Tondeur, Geometry of foliations, Monographs in Mathematics, 90. Birkhauser Verlag, Basel, 1997.

UNIVERSité DE METZ, ISGMP

E-mail address: benameur@math.univ-metz.fr

Mathematics, Statistics, and Computer Science, University of Illinois at Chicago

E-mail address: heitsch@math.uic.edu 